Statistica Neerlandica (1997) Vol. 51, nr. 3, pp. 318-344

\title{
Blackjack in Holland Casino's: basic, optimal and winning strategies
}

\author{
B. B. van der Genugten* \\ Department of Econometrics, Tilburg University, P.O. Box 90153, \\ 5000 LE Tilburg, The Netherlands
}

\begin{abstract}
This paper considers the card game Blackjack according to the rules of Holland Casino's in the Netherlands. Expected gains of strategies are derived with simulation and also with analytic tools. New efficiency concepts based on the gains of the basic and the optimal strategy are introduced. A general method for approximating expected gains for strategies based on card counting systems is developed. In particular it is shown how Thorp's Ten Count system and the High-Low system should be used in order to get positive expected gains. This implies that in Holland Casino's it is possible to beat the dealer in practice.
\end{abstract}

Key Words and Phrases: card games, optimality, card counting, Ten Count system, High-Low system.

\section{Introduction}

The card game of Blackjack (also known as Twenty One) is still today one of the most popular casino games. It has engendered much interest since by clever play it is possible for players to get an advantage over the house. This discovery was revealed in the sixties with the publication of the paper THORP (1960) and the subsequent famous book THORP (1966) entitled "Beat the Dealer". THORP showed that the player's expectation varies according to the undealt cards, and he indicated how to identify situations with a positive expectation. By raising the bet in such games an overall positive expected result can be obtained. Such winning strategies will beat the dealer in the long run.

However, casino's took their counter measures and changed the rules in order to get the advantage back. Today, these rules vary strongly between and even within casino's. For most of the variations it is still possible to obtain a serious advantage for professional hard working card counters. Although this mere fact seems to disturb casino boards terribly, the game is still attractive to exploit because most players are really amateurs and lose a lot of money. Another reason is that a winning strategy for one version of the game is a losing one for another variation.

There is a tremendous literature available on BJ (Blackjack). A lot of books are filled with strategy tables to use. Some of them are unreliable because they are based on rough approximating probability calculations; even the class of game variations for which they are supposed to be appropriate is not clearly indicated. The serious

\footnotetext{
* Ben.vdgenugten@kub.nl

(C) VVS, 1997. Published by Blackwell Publishers, 108 Cowley Road, Oxford OX4 1JF, UK and 350 Main Street, Malden, MA 02148, USA.
} 
ultimate guide for references is DALTON (1993). We mention here the easily available and reliable mathematical books and papers BALDwIN et al. (1956), EPSTEIN (1977), Gottlieb (1985), Griffin (1988), Chambliss and Roginski (1990), Yakowitz and Kollier (1992), and the appendix of BlACK (1993). All these publications deal exclusively with the American way of playing: with a dealer's hole card. In Europe in most casino's the game is played without a hole card.

The goal of this paper is to give a profound analysis of Blackjack as it is played in Holland Casino's in the Netherlands (Amsterdam, Breda, Eindhoven, Groningen, Nijmegen, Rotterdam, Scheveningen, Valkenburg, Zandvoort and Schiphol Airport). These BJHC-rules are exactly the same in all cities. They are typical Dutch in so far that the precise combination of the variations does not appear elsewhere. (We will describe the rules exactly in Section 2.)

A keystone for professional playing is the so called basic strategy. This strategy for BJHC was published first in VAN DER GENUGTEN (1993). Thereafter this strategy was revealed (and derived independently) by two Dutch professional Blackjack players Wind and WIND (1994).

In this paper we will analyse strategies for the BJHC-game and the concepts on which they are based. Utmost care is taken to give a clear definition of them since in literature this is often a source of confusion. Results are obtained with a special purpose computer package developed by the author.

Much of the material in this paper is, with minor changes, applicable to rules in other European casino's. For rules outside Europe differences are somewhat bigger due to the presence of the hole card.

This paper is a shortened version of VAN DER GENUGTEN (1995) obtained by omitting the description of the computer programs. It is organized as follows.

In Section 2 we give a description of the rules of BJHC.

In Section 3 we discuss the two components of strategies: the betfunction and the playing strategy. We formulate precisely the concept of optimality. This leads to a clear definition of the basic strategy. To fix the ideas we have also included its decision table in this section.

In Section 4 we consider the expected gains of strategies. By means of simulation we can give these gains for some naive strategies and the basic strategy. Also some rough estimates are given for the optimal strategy. We conclude this section by introducing efficiency concepts for arbitrary strategies.

The steady-state analysis in Section 5 makes clear which tools are needed for computer calculations for expected gains. These tools are indicated in the following two sections.

Section 6 describes the calculations for the optimal strategy and for given arbitrary strategies under the theoretical assumption that cards are drawn with replacement. In particular it treats the construction of the basic strategy. Section 7 describes the practical situation that cards are drawn without replacement.

In Section 8 a method is given for estimating the expected gains for optimal betting with arbitrary playing strategies, in particular for the basic strategy and the optimal strategy. This estimation method only gives crude estimates. 
In Section 9 we follow another approach by means of linear approximations of expected gains by card fractions. Also its relation to card counting systems for betting is described. Analytic results can be obtained by approximating the distribution of the running count by that of the Brownian bridge. More in detail TTC (Thorp's Ten Count) and HiLo (High-Low) are discussed.

Finally, in Section 10 we describe how the card counting systems of Section 9 can be used for playing decisions. Since optimal betting often involves maximal bets, high budgets are needed. Therefore we consider also some other betting concepts more suitable for low budget players. For readers only interested in practical strategies which beat the dealer this is the most interesting section.

\section{BJHC-rules}

In this section we will give a description of the BJHC-rules together with some notation to be used in the following. Game constants for which we will consider alternatives are presented as variables together with their standard values.

$\mathrm{BJHC}$ is a card game that is played with 2-7 players; mostly the number of players is $a=7$. The dealer, who is a member of the house, deals the cards out of a device called a shoe. A complete shoe consists of $n=6$ decks of playing cards of size 52 (therefore in total $k=52 n=312$ cards).

Cards are always dealt face up. So, at least in theory, every player can know the composition of the shoe at any stage of the game by observing the dealt cards.

Face cards have the value $10(T)$; non-face cards have their indicated value. An $A$ (ace) is counted as 1 or 11 depending on the other cards in the hand. If the sum of a hand with at least one ace counted as 11 would exceed 21 , then all aces are counted as 1 , otherwise one ace is counted as 11. A hand or sum is called soft if it contains an ace counted as 11; otherwise it is called hard. The main goal of players it to get hands with a sum as close as possible to but never exceeding 21 by drawing (asking the dealer for cards one after another) or standing (requesting no more cards) at the right moment. He busts (loses) if his (hard) sum exceeds 21. After all players the dealer draws cards too. He has no choice at all: he draws on sums $\leq 16$, stands on sums $\geq 17$ and $\leq 21$ (hard or soft), and busts (loses) on a (hard) sum $>21$. If a player and the dealer both stand, then the game is lost for the one holding the smallest sum. The combination $(A, T)$ is called "Blackjack" and beats any other sum of 21. Equals sums give a draw.

We code cards by their value and the ace by 1 . In general the card distribution in the shoe at a certain stage of the game is random and will be denoted by $C=(C(1), \ldots, C(10))$. Realizations will be denoted correspondingly with $c=(c(1), \ldots, c(10))$.

The playing stock $C_{1}$ for the first game is the (non-random) complete shoe $c_{0}=\left(k p_{1}, k p_{2}, \ldots, k p_{10}\right)=(4 n, 4 n, \ldots, 16 n)=(24,24, \ldots, 24,96)$, where $p_{1}=\cdots=p_{9}=1 / 13, p_{10}=4 / 13$ are the cards fractions in one deck. The remaining cards in the shoe after the first game become the (random) playing stock $C_{2}$ of the second game and so on. Used cards are placed into a discard rack. If during (or at the (c) VVS, 1997 
end of) a game the size $\Sigma C(i)$ of the current stock $C$ in the shoe decreases to a level equal to or less than $k(1-\lambda)$, then after this game the cards are reshuffled and the next game starts again with a complete shoe. In practice the fraction is marked by positioning a cut card in the shoe at about a played fraction $\lambda=2 / 3$ corresponding to a level of 104 remaining cards. However, in BJHC dealers are allowed to lower the cut card position to $\lambda=1 / 2$. This appears to be a disadvantage for the players and is only done when professional card counters join the game. We call a rowgame a whole sequence of games, from a complete shoe up to the game in which cut card falls or is reached.

At this moment the HC's in Amsterdam and Zandvoort are experimenting with card shuffling machines. After each game cards are automatically reshuffled. In this case a rowgame consists of exactly one game. If this reshuffling would be completely random, this would correspond to $\mathrm{BJ}$ with a fraction $\lambda=0$. (In practice there is a slight correlation between successive drawings.)

Outside the Netherlands there are still casino's which offer Blackjack without a cut card. This corresponds to a fraction $\lambda=1$. For that case, and also for other high values of $\lambda$, the shoe will get empty during a game. Then the cards in the discard rack are reshuffled and placed into the shoe for playing the remaining part of the game. In this paper we assume that then the next game is started with a reshuffled complete shoe. In BJHC the discard rack is never used for this purpose because the cut card position $\lambda$ is too small. However, for a general description and analysis it is worthful to consider the whole range $\lambda \in[0,1]$.

We describe in detail one game together with the decision points of the players.

The game starts with the betting of the players. The minimum and maximum bet can vary with the table. Today in BJHC the possible combinations (in Dutch guilders) are $(10,500),(20,1000),(40,1500)$ and in the "cercle privé" $(100,2500)$ (the combination $(5,500)$ in Scheveningen no longer exists). Fixing the minimum bet at the unit amount $B_{\min }=1$, the possible values of the maximum bets are $B_{\max }=50,37.5$ and 25 . Bets must be in the range $\left[1, B_{\max }\right]$.

After the players' betting round the dealer gives one card to each of the players and to himself (the dealercard). Then a second card is dealt to each of the players to make it a pair (not yet the dealer: no hole card). So at this stage all hands of players contain two cards.

If the dealercard is an $A$, every player may ask for insurance (IS) against a possible dealer's "Blackjack" later on. This is a side bet with an amount $\frac{1}{2} \times$ his original bet.

A player with the card combination "Blackjack" has to stand.

Next, players without "Blackjack", continue playing their hands, one after another, from player 1 to $a$.

If both cards of a hand have the same value, a player may split (SP) those cards and continue separately with two hands containing one card. To the additional hand a new bet equal to the original bet must be added. The first step in playing a split hand is that the dealer adds one new card to make it a pair. Repeated splitting is allowed without any restriction. However, with a no further split hand of two aces standing is obligatory. Pairs obtained with splitting cannot count as "Blackjack". 
If a pair (split or not) has a hard sum 9, 10 or 11 or a soft sum 19, 20 or 21 (not Blackjack), doubling down (DD) is permitted. Then the player doubles his original bet, draws exactly one card and has to stand thereafter. A soft sum becomes hard because every ace in this hand gets automatically the value 1 .

Finally, if a hand is not doubled, the player can draw or stand $(\mathrm{D} / \mathrm{S})$ as long as he did not stand or bust. Standing on a (hard or soft) 21 is obligatory. A non-split hand of three sevens gets a bonus of $1 \times$ the original bet.

After all players have played their hands the dealer draws cards for himself according to the fixed rule already indicated.

A winning player gains an amount $1 \times$ his original bet and even $1 \frac{1}{2} \times$ if he wins with "Blackjack". A losing player loses his bet. In case of a draw a player neither gains nor loses: his bet is returned.

If at least one player has taken insurance against a dealer's ace then, even in the case that no player stands, the dealer must draw at least one card to see if he gets "Blackjack". If he has "Blackjack" then the player gains $2 \times$ his insurance, otherwise he loses this insurance. Therefore, the dealer gives him immediately this gain and removes the player's cards from the table. This particular form of insurance is called evenmoney. (Of course, just for evenmoney the dealer would not draw a card.)

In the following we consider the number of decks $n$, the cut card position $\lambda$, the number of players $a$ and the maximum bet $B_{\max }$ as parameters. For the standard values $n=6, \lambda=2 / 3, a=7$ the playing time needed for one game is about 1 minute. Reshuffling takes 2 minutes. Since one rowgame contains approximately 10 games, this gives 12 minutes per rowgame or 5 rowgames per hour. So a professional player can play 10,000 rowgames (or 100,000 games) yearly if he works hard for 2000 hours per year. This should be kept in mind in judging expected gains per (row) game of strategies. For theoretical purposes concerning approximations we will also consider games in which every card is drawn with replacement. We refer to these games by the parameter values $n=\infty$ and $\lambda=0$. This implies that rowgames coincide with games.

\section{Strategies and optimality}

Consider a game with fixed parameters $n, a \lambda$ and $B_{\max }$. A strategy $\left(H_{v}, S_{v}\right)$ for a player $v$ consists of two parts: a betting strategy $H_{v}$ which prescribes the betsize at the start of each new game, and a playing strategy $S_{v}$ which prescribes the playing decisions IS, SP, DD, D/S at any stage of the game.

We restrict the class of all possible strategies of a player $v$ in the following way. His betsize at the start of a game shall only depend on the stock at that moment; therefore it can be characterized by a betfunction $H_{v}(c) \in\left[1, B_{\max }\right], c \in \mathcal{C}$, with $\mathcal{C}=\left\{c_{0}\right\} \cup\{c: \Sigma c(i)>k(1-\lambda)\}$ the class of possible stocks which can be encountered with betting.

The playing decisions of the player $v$ at a certain stage of the game shall only depend on the current or past stocks in that game and the exposed cards on the table at that stage. So a playing strategy $S_{v}$ is a function which specifies the playing (c) VVS, 1997 
decisions for every possible stock $c$ at the start of a game. More precisely, let $d_{0}(c)$ denote the sequence of the $2 a+1$ cards dealt by the dealer $(v=0)$ before the playing round starts, $d_{v}(c)$ (for $\left.v=0, \ldots, a\right)$ the whole sequence of cards used by the players $0, \ldots, v$ and, more specific, $d_{v j}=\left(d_{v-1}(c), x_{1 v}, \ldots, x_{j v}\right)$ the sequence up to the stage in which player $v$ already got additionally $j$ cards $x_{1 v}, \ldots, x_{j v}$. Then $S_{v}$ contains the relevant playing decisions at any stage $d_{v j}$ during this game. This constitutes a class $\mathcal{S}_{v}$ of playing strategies. The stocks during successive games only depend on the playing strategies $S_{v} \in \mathcal{S}_{v}$ for $v=1, \ldots, a$ of the players and not on their betfunctions. The restriction to such playing strategies gives no loss of generality at all.

Denote by $G_{1}(c)$ the (random) gain of a player $v$ for a game with starting stock $c \in \mathcal{C}$ and minimum bet $B_{\min }=1$. Then the (random) gain $G(c)$ of this player using the betfunction $H(c)$ is given by $G(c)=H(c) G_{1}(c), c \in C$.

For given playing strategies $S_{1}, \ldots, S_{a}$ the probability distribution $\mathcal{L}\left(G_{1}\right.$ instead of $(G c))$ is fixed. Given these strategies we call the betfunction $H_{v}$ of player $v$ optimal if it maximizes $E(G(c))$ for all $c \in \mathcal{C}$. Clearly, $H_{v}$ is optimal for

$$
H_{v}(c)= \begin{cases}1 & \text { if } E\left(G_{1}(c)\right) \leq 0 \\ B_{\max } & \text { if } E\left(G_{1}(c)\right)>0\end{cases}
$$

For fixed $S_{j}, j \neq v$, the distribution $\mathcal{L}\left(G_{1}(c)\right)$ only depends on the choice $S_{v} \in \mathcal{S}_{v}$. Given the $S_{j}$ with $j \neq v$ we call the playing strategy $S_{v}$ optimal if $S_{v}\left(d_{v j}\right)$ maximizes $E\left(G_{1}(c) \mid d_{v j}\right)$ for every stage $d_{v j}$ of the game that can be reached by player $v$ and for every stock $c \in \mathcal{C}$.

Optimality for player $v$ depends on the playing strategies $S_{j}$ of other players as well. In analyzing strategies for player $v$ we must make a specific choice for the playing strategies of the other players. A reasonable and pragmatic approach is to consider possible improvements of player $v$ amid other players of moderate skill playing independently of each other and following a simple so called basic strategy. Although in practice moderate players do not quite reach the level of this strategy, we choose it as a well defined reference point (see e.g. Bond, 1974, Keren and WAgenaAR, 1985, WagenaAr, 1988, Chau and Phillips, 1995).

We define the basic strategy $S_{\text {bas }}$ as the playing strategy which would be optimal under the theoretical assumption that all cards are drawn with replacement (i.e. the game with $n=\infty$ and $\lambda=0$ ). Clearly, under this assumption $E\left(G_{1}\left(c_{0}\right) \mid d_{v j}\right)$ will only depend on $d_{v j}$ through the dealercard and the cards in the hand(s) of player $v$ and not on the playing strategies $S_{j}$ of the other players $j \neq v$. Therefore $S_{\text {bas }}$ is the same for all players and can be tabulated as a function of the dealercard and characteristics of the player's hand. We describe its construction in Section 6. Table 1 gives the result.

So from now on while evaluating numerically the quality of the strategy of a particular player we assume that the other players follow the basic strategy $S_{\text {bas }}$. Therefore the optimal playing strategy $S_{\text {opt }}$ will only depend on the number of decks $n$, the number of players $a$, the cut card position $\lambda$ and the particular player $v$. We (c) VvS, 1997 
denote by $H_{\text {bas }}, H_{\text {opt }}$ the optimal betfunctions belonging to $S_{\text {bas }}, S_{\text {opt }}$, respectively. These functions depend on $B_{\max }$ too.

\section{Expected gains and efficiency}

Consider fixed parameters $n, a, \lambda$ and $B_{\max }$. For a fixed choice of playing strategies for each player, we consider the expected gain of a particular player with strategy $(H, S)$.

The random sequence of all successive stocks by dealing one card after another during the $m$ th game starting with stock $C_{m}$ and ending with $C_{m+1}$ determines the

Table 1. Basic Strategy $S_{\text {bas }}$ of BJHC

INSURANCE: never

SPLITTING $($ Split $=$ X; No Split $=-$ )

\begin{tabular}{lllllllllll}
\hline $\begin{array}{c}\text { Dealercard } \\
\text { Pair }\end{array}$ & $\mathrm{A}$ & 2 & 3 & 4 & 5 & 6 & 7 & 8 & 9 & $\mathrm{~T}$ \\
\hline AA & - & $\mathrm{X}$ & $\mathrm{X}$ & $\mathrm{X}$ & $\mathrm{X}$ & $\mathrm{X}$ & $\mathrm{X}$ & $\mathrm{X}$ & $\mathrm{X}$ & $\mathrm{X}$ \\
22 & - & $\mathrm{X}$ & $\mathrm{X}$ & $\mathrm{X}$ & $\mathrm{X}$ & $\mathrm{X}$ & $\mathrm{X}$ & - & - & - \\
33 & - & $\mathrm{X}$ & $\mathrm{X}$ & $\mathrm{X}$ & $\mathrm{X}$ & $\mathrm{X}$ & $\mathrm{X}$ & - & - & - \\
44 & - & - & - & - & $\mathrm{X}$ & $\mathrm{X}$ & - & - & - & - \\
55 & - & - & - & - & - & - & - & - & - & - \\
66 & - & $\mathrm{X}$ & $\mathrm{X}$ & $\mathrm{X}$ & $\mathrm{X}$ & $\mathrm{X}$ & - & - & - & - \\
77 & - & $\mathrm{X}$ & $\mathrm{X}$ & $\mathrm{X}$ & $\mathrm{X}$ & $\mathrm{X}$ & $\mathrm{X}$ & - & - & - \\
88 & - & $\mathrm{X}$ & $\mathrm{X}$ & $\mathrm{X}$ & $\mathrm{X}$ & $\mathrm{X}$ & $\mathrm{X}$ & $\mathrm{X}$ & $\mathrm{X}$ & - \\
99 & - & $\mathrm{X}$ & $\mathrm{X}$ & $\mathrm{X}$ & $\mathrm{X}$ & $\mathrm{X}$ & - & $\mathrm{X}$ & $\mathrm{X}$ & - \\
$\mathrm{TT}$ & - & - & - & - & - & - & - & - & - & - \\
\hline
\end{tabular}

DOUBLE DOWN $($ DDown $=\mathrm{X}$; No DDown $=-$ )

\begin{tabular}{lllllllllll}
\hline \multicolumn{1}{c}{ Dealercard } & $\mathrm{A}$ & 2 & 3 & 4 & 5 & 6 & 7 & 8 & 9 & $\mathrm{~T}$ \\
Sum & & & & & & & & & & \\
\hline Hard 9 & - & - & $\mathrm{X}$ & $\mathrm{X}$ & $\mathrm{X}$ & $\mathrm{X}$ & - & - & - & - \\
Hard 10 & - & $\mathrm{X}$ & $\mathrm{X}$ & $\mathrm{X}$ & $\mathrm{X}$ & $\mathrm{X}$ & $\mathrm{X}$ & $\mathrm{X}$ & $\mathrm{X}$ & - \\
Hard 11 & - & $\mathrm{X}$ & $\mathrm{X}$ & $\mathrm{X}$ & $\mathrm{X}$ & $\mathrm{X}$ & $\mathrm{X}$ & $\mathrm{X}$ & $\mathrm{X}$ & - \\
Soft 19-21 & - & - & - & - & - & - & - & - & - & - \\
\hline
\end{tabular}

DRAW/STAND $($ Draw $=\mathrm{X} ;$ Stand $=-$ )

\begin{tabular}{lllllllllll}
\hline \multicolumn{1}{c}{ Dealercard } & A & 2 & 3 & 4 & 5 & 6 & 7 & 8 & 9 & $\mathrm{~T}$ \\
Sum & & & & & & & & & & \\
\hline Hard $\leq 11$ & $\mathrm{X}$ & $\mathrm{X}$ & $\mathrm{X}$ & $\mathrm{X}$ & $\mathrm{X}$ & $\mathrm{X}$ & $\mathrm{X}$ & $\mathrm{X}$ & $\mathrm{X}$ & $\mathrm{X}$ \\
Hard 12 & $\mathrm{X}$ & $\mathrm{X}$ & $\mathrm{X}$ & - & - & - & $\mathrm{X}$ & $\mathrm{X}$ & $\mathrm{X}$ & $\mathrm{X}$ \\
Hard 13 & $\mathrm{X}$ & - & - & - & - & - & $\mathrm{X}$ & $\mathrm{X}$ & $\mathrm{X}$ & $\mathrm{X}$ \\
Hard 14 & $\mathrm{X}$ & - & - & - & - & - & $\mathrm{X}$ & $\mathrm{X}$ & $\mathrm{X}$ & $\mathrm{X}$ \\
Hard 15 & $\mathrm{X}$ & - & - & - & - & - & $\mathrm{X}$ & $\mathrm{X}$ & $\mathrm{X}$ & $\mathrm{X}$ \\
Hard 16 & $\mathrm{X}$ & - & - & - & - & - & $\mathrm{X}$ & $\mathrm{X}$ & $\mathrm{X}$ & $\mathrm{X}$ \\
Hard $\geq 17$ & - & - & - & - & - & - & - & - & - & - \\
Soft $\leq 17$ & $\mathrm{X}$ & $\mathrm{X}$ & $\mathrm{X}$ & $\mathrm{X}$ & $\mathrm{X}$ & $\mathrm{X}$ & $\mathrm{X}$ & $\mathrm{X}$ & $\mathrm{X}$ & $\mathrm{X}$ \\
Soft 18 & $\mathrm{X}$ & - & - & - & - & - & - & - & $\mathrm{X}$ & $\mathrm{X}$ \\
Soft $\geq 19$ & - & - & - & - & - & - & - & - & - & - \\
\hline
\end{tabular}

(C) VVS, 1997 
gain $G_{m}$ of the $m$ th game. Then the average gain $\mu_{\mathrm{G}}=\mu_{\mathrm{G}}(H, S)$ per game in the long run is given by

$$
\mu_{\mathrm{G}}=\lim _{m \rightarrow \infty} \frac{1}{m} \sum_{i=1}^{m} G_{i}, \quad \text { a.s. }
$$

(The average bet $\mu_{\mathrm{B}}=\mu_{\mathrm{B}}(H, S)$ per game in the long run is defined similarly.) Let $G R_{m}$ be the sum of all gains in the $m$ th rowgame and $N_{m}$ the number of games in this rowgame. Then the average gain $\mu_{\mathrm{GR}}$ and number of games $\mu_{\mathrm{N}}$ per rowgame is given by

$$
\left(\mu_{\mathrm{GR}}, \mu_{\mathrm{N}}\right)=\lim _{M \rightarrow \infty} \frac{1}{M} \sum_{m=1}^{M}\left(G R_{m}, N_{m}\right), \text { a.s. }
$$

Clearly,

$$
\mu_{\mathrm{G}}=\mu_{\mathrm{GR}} / \mu_{\mathrm{N}}
$$

For a given set of playing strategies the values of $\mu_{\mathrm{GR}}, \mu_{\mathrm{N}}$ and $\mu_{\mathrm{G}}$ can be determined with simulation. For a reasonable accuracy a simulation length of about $M=50,000,000$ rowgames is needed. On a PC-Pentium 90 such a simulation run requires 4 days.

In order to given an idea about the losses that are suffered with simple naive strategies we performed a simulation for BJHC with $a=7$ players, giving player $v$ the naive strategy "stand if sum $\geq v+11$ and draw otherwise". The strategy "stand for sum $\geq 12$ " means "never bust" and "stand for sum $\geq 17$ " is called "mimic the dealer". The betsize is 1 .

Table 2. Sim. gains of naive playing strategies $(n=6, \lambda=2 / 3, a=7, H \equiv 1)$ playing strategy: never IS, SP or DD; S if sum $\geq v+11$ and D otherwise ( $M=50,200,000$ rowgames $-\mu_{\mathrm{N}}=10.13$ games $)$

\begin{tabular}{llll}
\hline $\mathrm{P} v$ & $\mu_{\mathrm{GR}}$ & $\pm 95 \% C I$ & $\mu_{\mathrm{G}}\left(1, S_{v+11}\right)$ \\
\hline $\mathrm{D}$ & 4.622 & 0.004 & 0.4562 \\
$\mathrm{P} 5$ & -0.524 & 0.001 & -0.0517 \\
$\mathrm{P} 4$ & -0.527 & 0.001 & -0.0521 \\
$\mathrm{P} 6$ & -0.571 & 0.001 & -0.0564 \\
$\mathrm{P} 3$ & -0.586 & 0.001 & -0.0579 \\
$\mathrm{P} 2$ & -0.688 & 0.001 & -0.0679 \\
P1 & -0.814 & 0.001 & -0.0804 \\
$\mathrm{P} 7$ & -0.911 & 0.001 & -0.0899 \\
\hline
\end{tabular}

In the first column the player $\mathrm{D}$ refers to the dealer and $\mathrm{P} v$ to player $v$. The third column contains the half length of a $95 \%$ confidence interval for $\mu_{\mathrm{GR}}$. We see that these simple strategies lead to a disaster. Even the relatively best player P5 standing on 16 suffers a loss of more than $5 \%$. This is much more than a pure chance game such as Roulette would cost! Certainly such players are welcome at the Blackjack tables in HC. 
All players can do much better with a little bit more effort by following the basic strategy. Since the use of pencil and paper is strictly forbidden in BJHC, they just have to learn table 1 by heart. A simulation results is given in Table 3 .

Table 3. Sim. gains of $S_{\text {bas }}(n=6, \lambda=2 / 3, a=7, H \equiv 1)$

playing strategy: see Table 1

( $M=50,000,000$ rowgames $-\mu_{\mathrm{N}}=9.86$ games $)$

\begin{tabular}{llll}
\hline $\mathrm{P}$ & $\mu_{\mathrm{GR}}$ & $+95 \% C I$ & $\mu_{\mathrm{G}}\left(1, S_{\text {bas }}\right)$ \\
\hline $\mathrm{D}$ & 0.3726 & 0.005 & 0.0378 \\
$\mathrm{P} 5$ & -0.053 & 0.001 & -0.0054 \\
$\mathrm{P} 2$ & -0.053 & 0.001 & -0.0054 \\
$\mathrm{P} 6$ & -0.053 & 0.001 & -0.0054 \\
$\mathrm{P} 4$ & -0.053 & 0.001 & -0.0054 \\
$\mathrm{P} 7$ & -0.054 & 0.001 & -0.0054 \\
$\mathrm{P} 3$ & -0.054 & 0.001 & -0.0054 \\
$\mathrm{P} 1$ & -0.054 & 0.001 & -0.0055 \\
\hline
\end{tabular}

We see that $\mu_{\mathrm{G}}\left(1, S_{\mathrm{bas}}\right)=-0.0054$ is almost the same for all players and therefore independent of the position at the table. Although the value is still negative it is much higher than the values of $\mu_{\mathrm{G}}$ for the naive strategies in Table 2 .

The gain $\mu_{\mathrm{G}}\left(1, S_{\text {bas }}\right)$ for the basic strategy does hardly depend on the number of players. Table 4 gives the simulation result for 1 instead of 7 players.

Table 4. Sim. gains of $S_{\text {bas }}(n=6, \lambda=2 / 3, a=1, H \equiv 1)$

( $M=1,000,000,000$ rowgames $-\mu_{\mathrm{N}}=39.5$ games $)$

\begin{tabular}{llll}
\hline & $\mu_{\mathrm{GR}}$ & $\pm 95 \% C I$ & $\mu_{\mathrm{G}}\left(1, S_{\mathrm{bas}}\right)$ \\
\hline $\mathrm{P} 1$ & -0.217 & 0.001 & -0.0050 \\
\hline
\end{tabular}

The value -0.0050 differs slightly from $\mu_{\mathrm{G}}\left(1, S_{\mathrm{bas}}\right)=-0.0054$ for $n=6, \lambda=2 / 3$, $a=7$. Roughly speaking, the basic strategy with bet 1 gives a loss of 0.0050 to 0.0055 for all players and is independent of the number of players $a$.

Rather crude estimates of $\mu_{\mathrm{G}}$ can be given for the optimal betfunctions $H_{\mathrm{bas}}, H_{\mathrm{opt}}$ in combination with the playing strategies $S_{\text {bas }}, S_{\text {opt }}$. Table 5 gives some results for a particular player, thereby assuming that the other players play the basic strategy. (For details see VAN DER Genugten, 1995, sections 4 and 8.)

Table 5. Estimated gains $\left(n=6, \lambda=2 / 3, B_{\max }=50\right)$

\begin{tabular}{ll}
\hline Strategy & $\mu_{\mathrm{G}}$ \\
\hline$\left(1, S_{\text {bas }}\right)$ & -0.005 \\
$\left(1, S_{\text {opt }}\right)$ & -0.004 \\
$\left(H_{\text {bas }}, S_{\text {bas }}\right)$ & +0.08 \\
$\left(H_{\text {opt }}, S_{\text {opt }}\right)$ & +0.11 \\
\hline
\end{tabular}

The table shows that there exist strategies $(H, S)$ with positive expected gains. Using such strategies will beat the dealer in the long run. 
Consider for fixed playing strategies of the other players the strategy $(H, S)$ of a particular player. We define the total efficiency $\operatorname{TE}(H, S)$, the betting efficiency $\mathrm{BE}(H, S)$ and the (playing) strategy efficiency $\mathrm{SE}(H, S)=\mathrm{SE}(S)$ by, respectively,

$$
\begin{aligned}
\operatorname{TE}(H, S) & =\frac{\mu_{\mathrm{G}}(H, S)-\mu_{\mathrm{G}}\left(1, S_{\mathrm{bas}}\right)}{\mu_{\mathrm{G}}\left(H_{\mathrm{opt}}, S_{\mathrm{opt}}\right)-\mu_{\mathrm{G}}\left(1, S_{\mathrm{bas}}\right)} \\
\mathrm{BE}(H, S) & =\frac{\mu_{\mathrm{G}}(H, S)-\mu_{\mathrm{G}}(1, S)}{\mu_{\mathrm{G}}\left(H_{\mathrm{opt}}, S_{\mathrm{opt}}\right)-\mu_{\mathrm{G}}(1, S)} \\
\mathrm{SE}(S) & =\frac{\mu_{\mathrm{G}}(1, S)-\mu_{\mathrm{G}}\left(1, S_{\mathrm{bas}}\right)}{\mu_{\mathrm{G}}\left(1, S_{\mathrm{opt}}\right)-\mu_{\mathrm{G}}\left(1, S_{\mathrm{bas}}\right)}
\end{aligned}
$$

Clearly,

$$
\mathrm{TE}(H, S)=\mathrm{BE}(H, S)+T M \cdot \mathrm{SE}(S) \cdot(1-\mathrm{BE}(H, S))
$$

where TM is the table multiplier (not depending on $S$ ) defined by

$$
\mathrm{TM}=\frac{\mu_{\mathrm{G}}\left(1, S_{\mathrm{opt}}\right)-\mu_{\mathrm{G}}\left(1, S_{\mathrm{bas}}\right)}{\mu_{\mathrm{G}}\left(H_{\mathrm{opt}}, S_{\mathrm{opt}}\right)-\mu_{\mathrm{G}}\left(1, S_{\mathrm{bas}}\right)}
$$

For obtaining a high betting efficiency of the strategy $(H, S)$ we see that much effort should be put into the approximation $H$ of the optimal betfunction $H_{\text {bas }}$ in a simple playable way; the improvement of the playing strategy $S$ from $S_{\text {bas }}$ towards $S_{\text {opt }}$ is less important. This is even more true when the table multiplier TM is small. Then the total efficiency TE of $(H, S)$ is almost completely determined by its betting efficiency BE. So the improvement of $S$ towards $S_{\text {opt }}$ for influencing SE is of minor importance.

For $n=6$ and $B_{\max } \in[25,50], \lambda \in[1 / 2,2 / 3]$ the table multipliers TM of BJHC are in the range $0.01<\mathrm{TM}<0.03$ and therefore very small. (The figures in Table 5 are in agreement with this.) In fact the large number of decks $n=6$ has for a great deal reduced the effect of skill to betting.

Figure 6 shows the BE (betting efficiency) of $\left(H_{\mathrm{bas}}, S_{\mathrm{bas}}\right)$ for $1 / 6 \leq \lambda \leq 5 / 6$ and $B_{\max }=1,2,25,37.5$ and 50 . For $B_{\max }=1$ the efficiency is 0 by definition. For $B_{\max }=2$ the betting efficiency is very low (between 0.3 and 0.4 for varying values of $\lambda$. For the usual BJHC-values $B_{\max }=25,37.5$ and 50 the efficiency is more or less constant and only depends on $\lambda$. It decreases from 0.7 for $\lambda=0.3$ to 0.6 for $\lambda=0.8$. Clearly, for $S_{\text {bas }}$ it is much more important to use a good betfunction than improving $S_{\text {bas }}$ itself. (For details see VAN Der Genugten, 1995, section 8.)

\section{Steady-state analysis}

Consider a fixed choice of playing strategies. The random sequence $C_{1}, C_{2}, \ldots$ of starting stocks form an ergodic Markov chain with state space $\mathcal{C}$ and initial value $C_{1}=c_{0}$. Denote by

$$
\pi(c)=\lim _{m \rightarrow \infty} P\left\{C_{m}=c\right\}, \quad c \in \mathcal{C}
$$




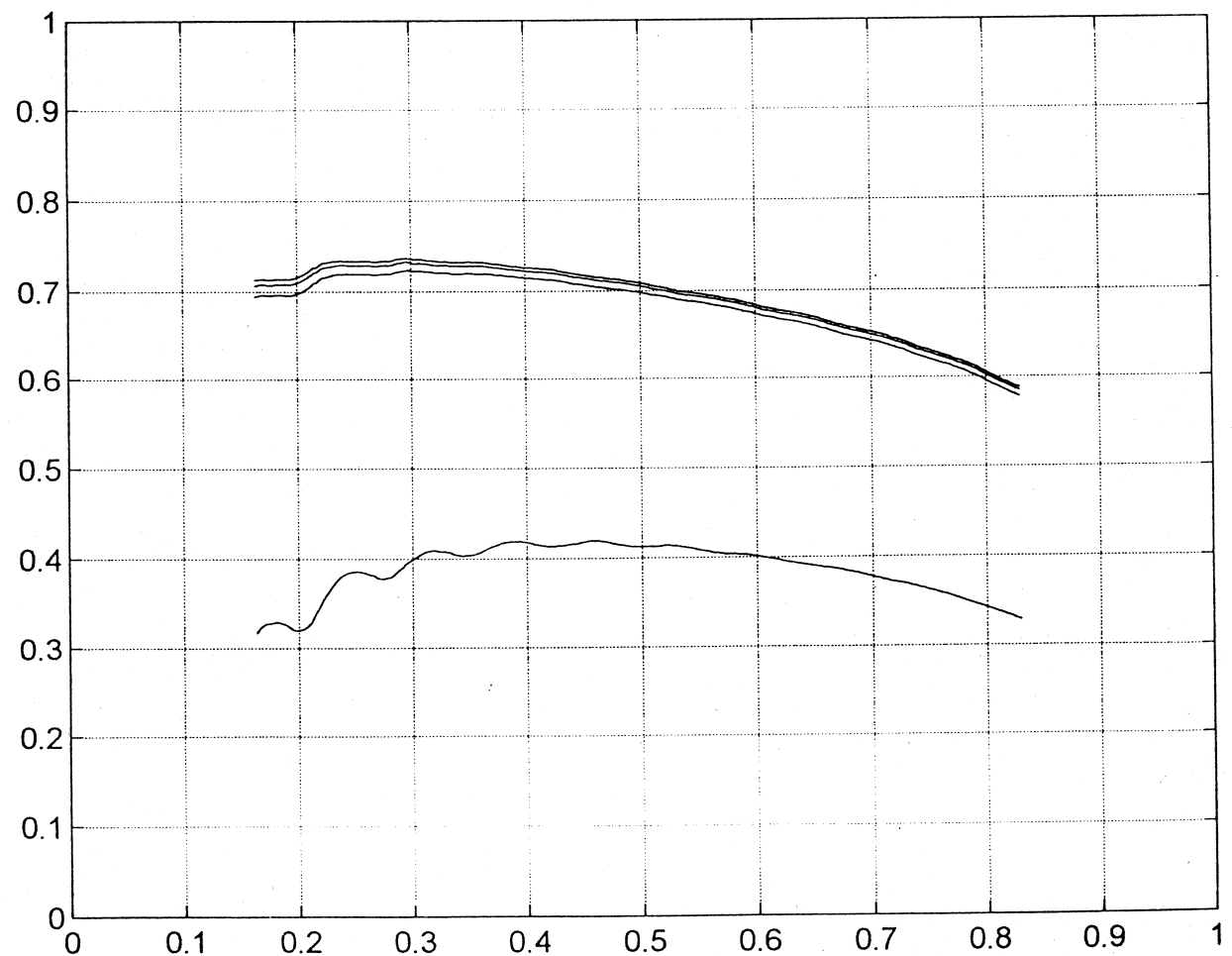

Fig. 6. $\mathrm{BE}\left(H_{\mathrm{bas}}, S_{\mathrm{bas}}\right)$ for $B_{\max }=1,2,25,37.5,50$ and for $1 / 6<\lambda<5 / 6(n=6, a=7)$.

its limit distribution (independent of $c_{0}$ ). We can express the average gains in the long run as expectations of gains in only one game if we start this game with a random stock $C_{1}=C$ with $\mathcal{L}(C)=\pi$ (the steady state). Then for $G_{1}=G_{1}(C)$ and $G=G(C)=H(C) G_{1}(C)$ we have according to the LLN for Markov chains:

$$
\mu_{\mathrm{G}_{1}}=E\left(G_{1}\right)=E\left(G_{1}(C)\right)=\sum_{c \in \mathrm{C}} \pi(c) E\left(G_{1}(c)\right)
$$

and more general,

$$
\mu_{\mathrm{G}}=E(G)=E\left(H(C) G_{1}(C)\right)=\sum_{c \in \mathrm{C}} \pi(c) H(c) E\left(G_{1}(c)\right)
$$

So, at least in theory, we can use (6) for calculating the expected gain $\mu_{\mathrm{G}}$ of any betfunction $H$ by determining $\pi(c)$ and $E\left(G_{1}(c)\right), c \in \mathcal{C}$.

In evaluating numerically the strategy of a particular player we taken for $\pi$ the limit probabilities for the assumed standard case that all players follows $S_{\text {bas }}$. So we neglect the effect that $\pi$ will change when the particular player deviates from $S_{\text {bas }}$. In practice this effect is small and good approximations will be obtained. Neglecting this effect, we see from (5) that the playing strategy $S_{\text {opt }}$ of a player as defined in Section 3 maximizes his $\mu_{\mathrm{G}_{1}}$. The corresponding betfunction $H_{\mathrm{opt}}$ (depending on $S_{\mathrm{opt}}$ ) (C) VVS, 1997 
maximizes his $\mu_{\mathrm{G}}$ in (6). The same holds for the optimal betfunction $H_{\mathrm{bas}}$ corresponding to $S_{\text {bas }}$.

For BJ with $a=1$ player we can calculate $E\left(G_{1}(c) \mid d_{1 j}\right)$ for every $c \in \mathcal{C}$ and for every card sequence $d_{1 j}$ of the player. This can be done not only for a given playing strategy but also for the optimal strategy. We distinguish the cases $n=\infty$ (drawing with replacement) and $n<\infty$ (drawing without replacement).

For $n=\infty$ the calculations are relatively simple because the card fractions in the stock remain unchanged. The computer programs solve the problems for a given stock $c$ in about $0.5 \mathrm{sec}$ on a PC-Pentium 90. We discuss the results in Section 6 .

For $n<\infty$ the calculations are very complicated since all possible stock developments from a given stock $c$ have to be taken into account. Yet, by a special coding system for such developments we were able to solve the problem within a finite time. However, the needed computer time for a given stock $c$ of moderate size with $n=6$ decks is about 5 days on the PC-Pentium 90 (and on a VAX mainframe still 19 hours). About $80 \%$ of the needed time is due to the calculations for repeated splitting. For many $c \in \mathcal{C}$ the differences between the values of $E\left(G_{1}(c)\right)$ for $n=\infty$ and moderate finite $n$ are small. This will be discussed in Section 7. Therefore in applying (6) we can take $n=\infty$ for approximations with values of $\lambda$ not too close to 1 .

In BJ with a number of players $a>1$, for a particular player $v$ there is also information contained in $d_{v-1}(c)$ and conditioning has to be performed for a whole sequence $d_{v j}$. This is simply impossible to do. However, the differences with $a=1$ player seem to be small. Therefore we will use the obtained results for one player also as approximations for the general case of a particular player among the other players.

With these approximations a straightforward computation of $\mu_{\mathrm{G}}$ by (6) is still impossible. The problem is the large number of stocks in $\mathcal{C}$ (about $(4 n+1)^{9}(16 n+1)$; for $n=6$ resulting in $3.7 \times 10^{14}$ ). Therefore we follow an approach which mixes simulation and analysis. Results are described in Section 8. They are based on conditioning to the fraction $t$ of played cards. This method is also used for the analysis of the card counting methods in Sections 9 and 10 .

\section{Expected gains for infinite decks}

In this section we assume that cards are drawn with replacement. Given a stock $c \in \mathcal{C}$ we can maximize $E\left(G_{1}(c) \mid d_{1 j}\right)$ for any sequence $d_{1 j}=\left(d_{0}(c), x_{11}, \ldots, x_{1 j}\right)$, where $d_{0}(c)$ contains the dealercard and the hands of two cards of all players and where $x_{11}, \ldots, x_{1 j}$ denotes the cards of the player thereafter. (Since all cards are drawn with replacement the stock at stage $d_{1 j}$ is still $c$.)

Table 7 gives the result for the starting stock $c_{0}$ for $n=6$. The unconditional mean becomes $E\left(G_{1}\left(c_{0}\right)\right)=-0.00614$. It is in fact an extension of Table 1 containing $S_{\text {bas }}$ since it optimizes decisions for the starting stock $c_{0}$. The main part has an entry for each dealercard $1, \ldots, 10$. Each hand has three columns:

Dec $=$ coded optimal decision,

Opt $=$ expected gains for the optimal decision,

Dif $=$ difference with the expected gain of the second best decision. 
Table 7. Optimal decisions and expected gains for the starting stock (with replacement)

Stock: 31224242424242424242496

GAME VALUE: -0.006144

(Decisions: $0=$ Stand $1=$ Draw $2=$ Double Down $3=$ Split $)$

INSURANCE

Decision: No - Opt: 0.000 - Dif: 0.038

\begin{tabular}{|c|c|c|c|c|c|c|c|c|c|c|c|}
\hline \multicolumn{2}{|c|}{ Dealer: } & 1 & 2 & 3 & 4 & 5 & 6 & 7 & 8 & 9 & $\mathrm{~T}$ \\
\hline \multicolumn{12}{|c|}{ SPLITTING } \\
\hline \multirow[t]{3}{*}{ A A } & Dec & 1 & 3 & 3 & 3 & 3 & 3 & 3 & 3 & 3 & 3 \\
\hline & Opt & -0.322 & 0.609 & 0.658 & 0.707 & 0.757 & 0.817 & 0.633 & 0.507 & 0.368 & 0.119 \\
\hline & Dif & 0.176 & 0.528 & 0.554 & 0.581 & 0.600 & 0.631 & 0.468 & 0.412 & 0.368 & 0.260 \\
\hline \multirow[t]{3}{*}{22} & Dec & 1 & 3 & 3 & 3 & 3 & 3 & 3 & 1 & 1 & 1 \\
\hline & Opt & -0.483 & -0.084 & -0.015 & 0.060 & 0.153 & 0.225 & 0.007 & -0.159 & -0.241 & -0.344 \\
\hline & Dif & 0.414 & 0.031 & 0.067 & 0.109 & 0.165 & 0.214 & 0.096 & 0.015 & 0.124 & 0.257 \\
\hline \multirow[t]{3}{*}{33} & Dec & 1 & 3 & 3 & 3 & 3 & 3 & 3 & 1 & 1 & 1 \\
\hline & Opt & -0.518 & -0.138 & -0.056 & 0.031 & 0.125 & 0.195 & -0.052 & -0.217 & -0.293 & -0.389 \\
\hline & Dif & 0.413 & 0.003 & 0.051 & 0.103 & 0.160 & 0.208 & 0.099 & 0.012 & 0.123 & 0.255 \\
\hline \multirow[t]{3}{*}{44} & Dec & 1 & 1 & 1 & 1 & 3 & 3 & 1 & 1 & 1 & 1 \\
\hline & Opt & -0.444 & -0.022 & 0.008 & 0.039 & 0.076 & 0.140 & 0.082 & -0.060 & -0.210 & -0.307 \\
\hline & Dif & 0.522 & 0.145 & 0.099 & 0.050 & 0.005 & 0.025 & 0.212 & 0.227 & 0.256 & 0.381 \\
\hline \multirow[t]{3}{*}{55} & Dec & 1 & 2 & 2 & 2 & 2 & 2 & 2 & 2 & 2 & 1 \\
\hline & Opt & -0.251 & 0.359 & 0.409 & 0.461 & 0.513 & 0.576 & 0.392 & 0.287 & 0.144 & -0.054 \\
\hline & Dif & 0.750 & 0.552 & 0.526 & 0.497 & 0.461 & 0.464 & 0.584 & 0.631 & 0.663 & 0.679 \\
\hline \multirow[t]{3}{*}{66} & Dec & 1 & 3 & 3 & 3 & 3 & 3 & 1 & 1 & 1 & 1 \\
\hline & Opt & -0.550 & -0.212 & -0.124 & -0.031 & 0.066 & 0.132 & -0.213 & -0.272 & -0.340 & -0.429 \\
\hline & Dif & 0.486 & 0.041 & 0.110 & 0.180 & 0.233 & 0.286 & 0.044 & 0.131 & 0.230 & 0.349 \\
\hline \multirow[t]{3}{*}{77} & Dec & 1 & 3 & 3 & 3 & 3 & 3 & 3 & 1 & 1 & 1 \\
\hline & Opt & -0.612 & -0.131 & -0.048 & 0.040 & 0.131 & 0.232 & -0.049 & -0.372 & -0.431 & -0.507 \\
\hline & Dif & 0.432 & 0.162 & 0.204 & 0.251 & 0.298 & 0.386 & 0.273 & 0.017 & 0.125 & 0.236 \\
\hline \multirow[t]{3}{*}{777} & Dec & 1 & 3 & 3 & 3 & 3 & 3 & 3 & 1 & 1 & 1 \\
\hline & Opt & -0.535 & -0.131 & -0.048 & 0.040 & 0.131 & 0.232 & -0.049 & -0.295 & -0.354 & -0.430 \\
\hline & Dif & 0.509 & 0.085 & 0.127 & 0.174 & 0.221 & 0.309 & 0.196 & 0.094 & 0.202 & 0.312 \\
\hline \multirow[t]{3}{*}{88} & Dec & 1 & 3 & 3 & 3 & 3 & 3 & 3 & 3 & 3 & 1 \\
\hline & Opt & -0.666 & 0.076 & 0.149 & 0.223 & 0.300 & 0.413 & 0.325 & -0.020 & -0.387 & -0.575 \\
\hline & Dif & 0.222 & 0.369 & 0.401 & 0.434 & 0.467 & 0.566 & 0.740 & 0.438 & 0.123 & 0.039 \\
\hline \multirow[t]{3}{*}{99} & Dec & 0 & 3 & 3 & 3 & 3 & 3 & 3 & 3 & 3 & 0 \\
\hline & Opt & -0.377 & 0.196 & 0.259 & 0.324 & 0.393 & 0.472 & 0.400 & 0.235 & -0.077 & -0.242 \\
\hline & Dif & 0.329 & 0.074 & 0.111 & 0.148 & 0.194 & 0.189 & 0.030 & 0.129 & 0.106 & 0.195 \\
\hline \multirow[t]{3}{*}{$\mathrm{T} \mathrm{T}$} & Dec & 0 & 0 & 0 & 0 & 0 & 0 & 0 & 0 & 0 & 0 \\
\hline & Opt & 0.146 & 0.640 & 0.650 & 0.661 & 0.670 & 0.704 & 0.773 & 0.792 & 0.758 & 0.435 \\
\hline & Dif & 0.649 & 0.275 & 0.238 & 0.200 & 0.158 & 0.128 & 0.259 & 0.396 & 0.525 & 0.542 \\
\hline
\end{tabular}

DOUBLE DOWN

$\begin{array}{llrlllllllll}\text { H } 9 & \text { Dec } & 1 & 1 & 2 & 2 & 2 & 2 & 1 & 1 & 1 & 1 \\ & \text { Opt } & -0.353 & 0.074 & 0.121 & 0.182 & 0.243 & 0.317 & 0.172 & 0.098 & -0.052 & -0.218 \\ & \text { Dif } & 0.562 & 0.013 & 0.020 & 0.053 & 0.085 & 0.121 & 0.068 & 0.125 & 0.249 & 0.367 \\ \text { H10 } & \text { Dec } & 1 & 2 & 2 & 2 & 2 & 2 & 2 & 2 & 2 & 1 \\ & \text { Opt } & -0.251 & 0.359 & 0.409 & 0.461 & 0.513 & 0.576 & 0.392 & 0.287 & 0.144 & -0.054 \\ & \text { Dif } & 0.374 & 0.176 & 0.203 & 0.230 & 0.256 & 0.288 & 0.136 & 0.089 & 0.028 & 0.108 \\ \text { H11 } & \text { Dec } & 1 & 2 & 2 & 2 & 2 & 2 & 2 & 2 & 2 & 1 \\ & \text { Opt } & -0.209 & 0.471 & 0.518 & 0.566 & 0.615 & 0.667 & 0.463 & 0.351 & 0.228 & 0.033 \\ & \text { Dif } & 0.331 & 0.232 & 0.257 & 0.283 & 0.307 & 0.334 & 0.171 & 0.121 & 0.070 & 0.021\end{array}$


Table 7-continued

\begin{tabular}{llllllllllll}
\hline Dealer: & & 1 & 2 & 3 & 4 & 5 & 6 & 7 & 8 & 9 & T \\
\hline S19 & Dec & 0 & 0 & 0 & 0 & 0 & 0 & 0 & 0 & 0 & 0 \\
& Opt & -0.115 & 0.386 & 0.404 & 0.423 & 0.440 & 0.496 & 0.616 & 0.594 & 0.288 & -0.019 \\
& Dif & 0.800 & 0.325 & 0.284 & 0.241 & 0.196 & 0.179 & 0.512 & 0.620 & 0.589 & 0.566 \\
S20 & Dec & 0 & 0 & 0 & 0 & 0 & 0 & 0 & 0 & 0 & 0 \\
& Opt & 0.146 & 0.640 & 0.650 & 0.661 & 0.670 & 0.704 & 0.773 & 0.792 & 0.758 & 0.435 \\
& Dif & 0.771 & 0.281 & 0.241 & 0.200 & 0.158 & 0.128 & 0.381 & 0.505 & 0.614 & 0.597 \\
S21 & Dec & 0 & 0 & 0 & 0 & 0 & 0 & 0 & 0 & 0 & 0 \\
& Opt & 0.331 & 0.882 & 0.885 & 0.889 & 0.892 & 0.903 & 0.926 & 0.931 & 0.939 & 0.812 \\
& Dif & 0.871 & 0.411 & 0.368 & 0.323 & 0.277 & 0.235 & 0.463 & 0.580 & 0.711 & 0.800
\end{tabular}

DRAW/STAND

\begin{tabular}{|c|c|c|c|c|c|c|c|c|c|c|c|}
\hline \multirow[t]{3}{*}{ H 3} & Dec & 1 & 1 & 1 & 1 & 1 & 1 & 1 & 1 & 1 & 1 \\
\hline & $\mathrm{Opt}$ & -0.465 & -0.101 & -0.069 & -0.036 & 0.000 & 0.024 & -0.057 & -0.131 & -0.215 & -0.322 \\
\hline & Dif & 0.304 & 0.192 & 0.183 & 0.175 & 0.167 & 0.178 & 0.418 & 0.380 & 0.328 & 0.254 \\
\hline \multirow[t]{3}{*}{ H 4} & Dec & 1 & 1 & 1 & 1 & 1 & 1 & 1 & 1 & 1 & 1 \\
\hline & $\mathrm{Opt}$ & -0.483 & -0.115 & -0.083 & -0.049 & -0.012 & 0.011 & -0.088 & -0.159 & -0.241 & -0.344 \\
\hline & Dif & 0.287 & 0.178 & 0.170 & 0.162 & 0.155 & 0.165 & 0.387 & 0.351 & 0.302 & 0.232 \\
\hline \multirow[t]{3}{*}{ H 5} & Dec & 1 & 1 & 1 & 1 & 1 & 1 & 1 & 1 & 1 & 1 \\
\hline & Opt & -0.501 & -0.128 & -0.095 & -0.061 & -0.024 & -0.001 & -0.119 & -0.188 & -0.267 & -0.366 \\
\hline & Dif & 0.269 & 0.165 & 0.157 & 0.150 & 0.143 & 0.153 & 0.356 & 0.322 & 0.277 & 0.210 \\
\hline \multirow[t]{3}{*}{ H 6} & Dec & 1 & 1 & 1 & 1 & 1 & 1 & 1 & 1 & 1 & 1 \\
\hline & Opt & -0.518 & -0.141 & -0.107 & -0.073 & -0.035 & -0.013 & -0.152 & -0.217 & -0.293 & -0.389 \\
\hline & Dif & 0.251 & 0.152 & 0.145 & 0.138 & 0.132 & 0.141 & 0.323 & 0.293 & 0.251 & 0.187 \\
\hline \multirow[t]{3}{*}{ H 7} & Dec & 1 & 1 & 1 & 1 & 1 & 1 & 1 & 1 & 1 & 1 \\
\hline & Opt & -0.522 & -0.109 & -0.077 & -0.043 & -0.007 & 0.029 & -0.069 & -0.211 & -0.285 & -0.371 \\
\hline & Dif & 0.247 & 0.184 & 0.176 & 0.168 & 0.160 & 0.183 & 0.407 & 0.300 & 0.258 & 0.204 \\
\hline \multirow[t]{3}{*}{ H 8} & Dec & 1 & 1 & 1 & 1 & 1 & 1 & 1 & 1 & 1 & 1 \\
\hline & $\mathrm{Opt}$ & -0.444 & -0.022 & 0.008 & 0.039 & 0.071 & 0.115 & 0.082 & -0.060 & -0.210 & -0.307 \\
\hline & Dif & 0.325 & 0.271 & 0.260 & 0.250 & 0.238 & 0.269 & 0.558 & 0.451 & 0.333 & 0.269 \\
\hline \multirow[t]{3}{*}{ H 9} & Dec & 1 & 1 & 1 & 1 & 1 & 1 & 1 & 1 & 1 & 1 \\
\hline & Opt & -0.353 & 0.074 & 0.101 & 0.129 & 0.158 & 0.196 & 0.172 & 0.098 & -0.052 & -0.218 \\
\hline & Dif & 0.416 & 0.367 & 0.354 & 0.340 & 0.325 & 0.350 & 0.647 & 0.609 & 0.491 & 0.358 \\
\hline \multirow[t]{3}{*}{$\mathrm{H} 10$} & Dec & 1 & 1 & 1 & 1 & 1 & 1 & 1 & 1 & 1 & 1 \\
\hline & Opt & -0.251 & 0.182 & 0.206 & 0.230 & 0.256 & 0.288 & 0.257 & 0.198 & 0.117 & -0.054 \\
\hline & Dif & 0.518 & 0.475 & 0.458 & 0.442 & 0.423 & 0.441 & 0.732 & 0.708 & 0.660 & 0.522 \\
\hline \multirow[t]{3}{*}{ H11 } & Dec & 1 & 1 & 1 & 1 & 1 & 1 & 1 & 1 & 1 & 1 \\
\hline & $\mathrm{Opt}$ & -0.209 & 0.238 & 0.260 & 0.283 & 0.307 & 0.334 & 0.292 & 0.230 & 0.158 & 0.033 \\
\hline & Dif & 0.561 & 0.531 & 0.513 & 0.494 & 0.475 & 0.487 & 0.768 & 0.740 & 0.701 & 0.609 \\
\hline \multirow[t]{3}{*}{ H12 } & Dec & 1 & 1 & 1 & 0 & 0 & 0 & 1 & 1 & 1 & 1 \\
\hline & Opt & -0.550 & -0.253 & -0.234 & -0.211 & -0.167 & -0.154 & -0.213 & -0.272 & -0.340 & -0.429 \\
\hline & Dif & 0.219 & 0.039 & 0.019 & 0.002 & 0.026 & 0.017 & 0.263 & 0.239 & 0.203 & 0.147 \\
\hline \multirow[t]{3}{*}{ H13 } & Dec & 1 & 0 & 0 & 0 & 0 & 0 & 1 & 1 & 1 & 1 \\
\hline & Opt & -0.582 & -0.293 & -0.252 & -0.211 & -0.167 & -0.154 & -0.269 & -0.324 & -0.387 & -0.469 \\
\hline & Dif & 0.187 & 0.015 & 0.039 & 0.063 & 0.090 & 0.082 & 0.206 & 0.187 & 0.156 & 0.106 \\
\hline \multirow[t]{3}{*}{ H14 } & Dec & 1 & 0 & 0 & 0 & 0 & 0 & 1 & 1 & 1 & 1 \\
\hline & $\mathrm{Opt}$ & -0.612 & -0.293 & -0.252 & -0.211 & -0.167 & -0.154 & -0.321 & -0.372 & -0.431 & -0.507 \\
\hline & Dif & 0.157 & 0.069 & 0.096 & 0.124 & 0.154 & 0.147 & 0.154 & 0.139 & 0.112 & 0.068 \\
\hline \multirow[t]{3}{*}{777} & Dec & 1 & 1 & 1 & 1 & 1 & 1 & 1 & 1 & 1 & 1 \\
\hline & $\mathrm{Opt}$ & -0.535 & -0.216 & -0.175 & -0.134 & -0.090 & -0.077 & -0.244 & -0.295 & -0.354 & -0.430 \\
\hline & Dif & 0.234 & 0.077 & 0.077 & 0.077 & 0.077 & 0.077 & 0.231 & 0.216 & 0.189 & 0.145 \\
\hline
\end{tabular}


Table 7-continued

\begin{tabular}{|c|c|c|c|c|c|c|c|c|c|c|c|}
\hline Dealer: & & 1 & 2 & 3 & 4 & 5 & 6 & 7 & 8 & 9 & $\mathrm{~T}$ \\
\hline \multirow[t]{3}{*}{ H15 } & Dec & 1 & 0 & 0 & 0 & 0 & 0 & 1 & 1 & 1 & 1 \\
\hline & Opt & -0.640 & -0.293 & -0.252 & -0.211 & -0.167 & -0.154 & -0.370 & -0.417 & -0.472 & -0.543 \\
\hline & Dif & 0.129 & 0.124 & 0.154 & 0.185 & 0.218 & 0.212 & 0.106 & 0.094 & 0.072 & 0.033 \\
\hline \multirow[t]{3}{*}{ H16 } & Dec & 1 & 0 & 0 & 0 & 0 & 0 & 1 & 1 & 1 & 1 \\
\hline & Opt & -0.666 & -0.293 & -0.252 & -0.211 & -0.167 & -0.154 & -0.415 & -0.458 & -0.509 & -0.575 \\
\hline & Dif & 0.104 & 0.178 & 0.212 & 0.245 & 0.282 & 0.277 & 0.061 & 0.052 & 0.034 & 0.001 \\
\hline \multirow[t]{3}{*}{ H17 } & Dec & 0 & 0 & 0 & 0 & 0 & 0 & 0 & 0 & 0 & 0 \\
\hline & Opt & -0.639 & -0.153 & -0.117 & -0.081 & -0.045 & 0.012 & -0.107 & -0.382 & -0.423 & -0.464 \\
\hline & Dif & 0.055 & 0.383 & 0.414 & 0.446 & 0.478 & 0.520 & 0.377 & 0.124 & 0.131 & 0.152 \\
\hline \multirow[t]{3}{*}{ H18 } & Dec & 0 & 0 & 0 & 0 & 0 & 0 & 0 & 0 & 0 & 0 \\
\hline & Opt & -0.377 & 0.122 & 0.148 & 0.176 & 0.200 & 0.283 & 0.400 & 0.106 & -0.183 & -0.242 \\
\hline & Dif & 0.364 & 0.744 & 0.768 & 0.793 & 0.815 & 0.891 & 0.991 & 0.697 & 0.433 & 0.433 \\
\hline \multirow[t]{3}{*}{ H19 } & Dec & 0 & 0 & 0 & 0 & 0 & 0 & 0 & 0 & 0 & 0 \\
\hline & Opt & -0.155 & 0.386 & 0.404 & 0.423 & 0.440 & 0.496 & 0.616 & 0.594 & 0.288 & -0.019 \\
\hline & Dif & 0.694 & 1.115 & 1.132 & 1.150 & 1.166 & 1.219 & 1.331 & 1.308 & 1.003 & 0.732 \\
\hline \multirow[t]{3}{*}{$\mathrm{H} 20$} & Dec & 0 & 0 & 0 & 0 & 0 & 0 & 0 & 0 & 0 & 0 \\
\hline & Opt & 0.146 & 0.640 & 0.650 & 0.661 & 0.670 & 0.704 & 0.773 & 0.792 & 0.758 & 0.435 \\
\hline & Dif & 1.044 & 1.495 & 1.505 & 1.516 & 1.525 & 1.558 & 1.625 & 1.643 & 1.609 & 1.296 \\
\hline \multirow[t]{3}{*}{$\mathrm{S} 12$} & Dec & 1 & 1 & 1 & 1 & 1 & 1 & 1 & 1 & 1 & 1 \\
\hline & Opt & -0.322 & 0.082 & 0.104 & 0.127 & 0.156 & 0.186 & 0.165 & 0.095 & 0.000 & -0.142 \\
\hline & Dif & 0.448 & 0.375 & 0.356 & 0.338 & 0.324 & 0.340 & 0.641 & 0.606 & 0.543 & 0.434 \\
\hline \multirow[t]{3}{*}{$\mathrm{S} 13$} & Dec & 1 & 1 & 1 & 1 & 1 & 1 & 1 & 1 & 1 & 1 \\
\hline & Opt & -0.347 & 0.047 & 0.074 & 0.102 & 0.133 & 0.162 & 0.122 & 0.054 & -0.038 & -0.174 \\
\hline & Dif & 0.422 & 0.339 & 0.326 & 0.314 & 0.301 & 0.315 & 0.598 & 0.565 & 0.505 & 0.402 \\
\hline \multirow[t]{3}{*}{ S14 } & Dec & 1 & 1 & 1 & 1 & 1 & 1 & 1 & 1 & 1 & 1 \\
\hline & Opt & -0.373 & 0.022 & 0.051 & 0.080 & 0.112 & 0.139 & 0.080 & 0.013 & -0.075 & -0.206 \\
\hline & Dif & 0.397 & 0.315 & 0.303 & 0.291 & 0.279 & 0.293 & 0.555 & 0.524 & 0.468 & 0.370 \\
\hline \multirow[t]{3}{*}{$\mathrm{S} 15$} & Dec & 1 & 1 & 1 & 1 & 1 & 1 & 1 & 1 & 1 & 1 \\
\hline & Opt & -0.398 & -0.000 & 0.029 & 0.059 & 0.092 & 0.118 & 0.037 & -0.027 & -0.112 & -0.237 \\
\hline & Dif & 0.372 & 0.293 & 0.281 & 0.270 & 0.259 & 0.272 & 0.512 & 0.483 & 0.431 & 0.339 \\
\hline \multirow[t]{3}{*}{ S16 } & Dec & 1 & 1 & 1 & 1 & 1 & 1 & 1 & 1 & 1 & 1 \\
\hline & Opt & -0.422 & -0.021 & 0.009 & 0.040 & 0.073 & 0.099 & -0.005 & -0.067 & -0.149 & -0.268 \\
\hline & Dif & 0.347 & 0.272 & 0.261 & 0.251 & 0.241 & 0.253 & 0.470 & 0.444 & 0.395 & 0.307 \\
\hline \multirow[t]{3}{*}{ S17 } & Dec & 1 & 1 & 1 & 1 & 1 & 1 & 1 & 1 & 1 & 1 \\
\hline & Opt & -0.432 & -0.000 & 0.029 & 0.059 & 0.091 & 0.128 & 0.054 & -0.073 & -0.150 & -0.259 \\
\hline & Dif & 0.207 & 0.152 & 0.146 & 0.140 & 0.136 & 0.116 & 0.161 & 0.309 & 0.273 & 0.206 \\
\hline \multirow[t]{3}{*}{ S18 } & Dec & 1 & 0 & 0 & 0 & 0 & 0 & 0 & 0 & 1 & 1 \\
\hline & Opt & -0.372 & 0.122 & 0.148 & 0.176 & 0.200 & 0.283 & 0.400 & 0.106 & -0.101 & -0.210 \\
\hline & Dif & 0.005 & 0.059 & 0.058 & 0.057 & 0.052 & 0.093 & 0.229 & 0.066 & 0.082 & 0.032 \\
\hline \multirow[t]{3}{*}{ S19 } & Dec & 0 & 0 & 0 & 0 & 0 & 0 & 0 & 0 & 0 & 0 \\
\hline & Opt & -0.115 & 0.386 & 0.404 & 0.423 & 0.440 & 0.496 & 0.616 & 0.594 & 0.288 & -0.019 \\
\hline & Dif & 0.196 & 0.262 & 0.255 & 0.248 & 0.237 & 0.256 & 0.395 & 0.442 & 0.280 & 0.140 \\
\hline \multirow[t]{3}{*}{ S20 } & Dec & 0 & 0 & 0 & 0 & 0 & 0 & 0 & 0 & 0 & 0 \\
\hline & Opt & 0.146 & 0.640 & 0.650 & 0.661 & 0.670 & 0.704 & 0.773 & 0.792 & 0.758 & 0.435 \\
\hline & Dif & 0.397 & 0.457 & 0.444 & 0.431 & 0.414 & 0.416 & 0.516 & 0.594 & 0.642 & 0.489 \\
\hline \multicolumn{12}{|c|}{ GAIN FOR SUM = 21} \\
\hline & BJ & 1.038 & 1.500 & 1.500 & 1.500 & 1.500 & 1.500 & 1.500 & 1.500 & 1.500 & 1.385 \\
\hline & NoBJ & 0.331 & 0.882 & 0.885 & 0.889 & 0.892 & 0.903 & 0.926 & 0.931 & 0.939 & 0.812 \\
\hline
\end{tabular}


(Note that during the game "splitting" comes before "double down", and "double down" before "draw/stand".) For example, with a hand $(5,5)$ against a dealercard 8 we see under "splitting" Dec $=2$. So we should not split but double down. In this case the expected gain is Opt $=0.287$. Splitting would give a difference $\mathrm{Dif}=0.631$ compared with the optimal decision, leading to an expected gain of $0.287-$ $0.631=-0.344$. The sum of $(5,5)$ is $\mathrm{H}(\operatorname{ard}) 10$. Under "double down" for $\mathrm{H} 10$ we get the same value $\mathrm{Opt}=0.287$. The second best decision (draw or stand) has a difference Dif $=0.089$, leading to an expected gain $0.287-0.089=0.198$ for "not double down". Under "draw/stand" we find that this value corresponds to Dec $=1$ (drawing). The difference is 0.708 leading to an expected gain of $0.198-0.708=$ -0.510 for standing.

Under "splitting" and "draw/stand" the code 777 refers to the situation that the extra bonus for three sevens can be obtained and 77 or H14 to the situation that this is not the case.

For $c \neq c_{0}$ the optimal decisions can be quite different from those of $S_{\text {bas }}$.

We will not describe the algorithms leading to the results above. Only the algorithm for insurance is very easy. Let $f_{10}=c(10) / \Sigma c(i)$ be the fraction of tens in the current stock $c$. This equals the probability that the dealer gets BJ. Therefore the expected gain with insurance is $-\frac{1}{2}+\frac{3}{2} f_{10}$. So we should insure if $f_{10}>\frac{1}{3}$. For the starting stock $c_{0}$ we have $f_{10}=p_{10}<\frac{1}{3}$. Therefore the basic strategy prescribes "never insure".

For a discussion of the other algorithms we refer to VAN DeR GenUGTen (1995), section 6 .

\section{Expected gains for finite decks}

In this section we assume that cards are drawn without replacement. We have to maximize $E\left(G_{1}(c) \mid d_{1 j}\right)$ for any sequence $d_{1 j}=\left(d_{0}(c), x_{11}, \ldots, x_{1 j}\right)$ for $a=1$ player.

For the unconditional mean with $n=6$ decks we find $E\left(G_{1}\left(c_{0}\right)\right)=-0.0052$. For the infinite case $n=\infty$ we found the value -0.0061 (see Section 6). The difference is mainly due to the different procedures in drawing cards.

Intermediate results are the expected gains $E\left(G_{1}\left(c_{0}\right) \mid d_{0}\left(c_{0}\right)\right)$ given the dealercard and the hand of two cards of the player. So these three cards are removed from the starting stock before the calculations for splitting, double down and draw/stand are made. It is interesting to compare these results with the corresponding infinite case. It appears that the optimal decisions for $n=6$ and $n=\infty$ coincide for almost all players' hands. There are some exceptions, e.g. for $n=6$ we should draw for $(10,2)$ and stand for all other hands against a dealercard 4. However, the effect on the expected gain is very small (For details we refer to VAN DER Genugten, 1995, section 7.)

There is a systematic pattern in the unconditional mean for varying $n$. Table 8 contains maximal expected gains for varying $n$. The table shows that $S_{\text {opt }}$ (or $S_{\text {bas }}$ ) gives a higher expected gain when the number of decks $n$ decreases, although for $n \geq 1$ the value is still negative. 
Table 8. Expected gains $E\left(G_{1}\left(c_{0}\right)\right)$ for $S_{\text {opt }}(a=1)$

\begin{tabular}{rrrrrrrr}
\hline$n$ & $E\left(G_{1}\left(c_{0}\right)\right)$ & $n$ & $E\left(G_{1}\left(c_{0}\right)\right)$ & $n$ & $E\left(G_{1}\left(c_{0}\right)\right)$ & $n$ & $E\left(G_{1}\left(c_{0}\right)\right)$ \\
\hline$\infty$ & -0.0061 & 20 & -0.0059 & 4 & -0.0047 & 1 & -0.0029 \\
100 & -0.0061 & 6 & -0.0052 & 3 & -0.0043 & $\frac{1}{2}$ & +0.0071 \\
50 & -0.0060 & 5 & -0.0050 & 2 & -0.0033 & & \\
\hline
\end{tabular}

\section{Estimation of expected gains}

Consider a fixed choice of playing strategies and one particular player. We continue the steady-state analysis in Section 5 by conditioning to the fraction $t$ of played cards. Let $\mathcal{C}_{t}=\{c: \Sigma c(j)=k-k t\}$ denote the set of stocks containing $k-k t$ cards $(t=0,1 / k, \ldots, \lambda-1 / k)$. Then from (6) we get

$$
\mu_{G}=\sum_{t=0}^{\lambda-1 / k} p(t) E_{t}(G)
$$

with

$$
\begin{aligned}
p(t) & =P\left\{C \in \mathcal{C}_{\mathrm{t}}\right\} \\
E_{t}(G) & =E_{t}\left(H(C) G_{1}(C)\right)
\end{aligned}
$$

Here $E_{t}$ denotes the conditional expectation given $\left\{C \in \mathcal{C}_{t}\right\}$. In particular, for the optimal betfunction $H$ corresponding to the chosen playing strategy we get

$$
\left.E_{t}(G)=E_{t}\left(G_{1}(C)\right)+\left(B_{\max }-1\right) E_{t}\left\{G_{1}(C)\right) I\left(G_{1}(C)>0\right)\right\}
$$

Note that substitution of $B_{\max }=1$ gives the result for the unit betfunction $H=1$.

By giving all players the basic strategy $S_{\text {bas }}$, the probabilities $p(t)$ can be determined by simulation. Figure 9 gives a graphical presentation of $p(t) / p(0)$ for $0<t<1$ for $n=6, \lambda=1, a=7$ based on a simulation of $M=40,000,000$ rowgames.

Note the oscillating pattern due to the fact that every rowgame starts anew with the same starting stock $c_{0}$. From the $p(t)$ for $\lambda=1$ we easily get the $p(t)$ for arbitrary $\lambda$ by truncation and rescaling. As mentioned at the end of Section 5 we cannot calculate (7) from (9) due to the fact that the calculation of $E\left(G_{1}(c)\right)$ for all $c$ is too time consuming. For optimal betting we used simulation of a restricted size to get for all $t$ an approximation of the conditional expectations $E_{t}\left(G_{1}(C)\right)$ and $E_{t}\left(G_{1}(C) I\left(G_{1}(C)>0\right)\right)$ appearing in $(10)$. We did this for the basic strategy $S_{\text {bas }}$ as well as the optimal playing strategy $S_{\text {opt }}$. With (7) this leads to the corresponding expected gains $\mu_{\mathrm{G}}$ for $\left(H_{\mathrm{bas}}, S_{\mathrm{bas}}\right)$ and $\left(H_{\mathrm{opt}}, S_{\mathrm{opt}}\right)$. The results have already been given in Table 5. (For details see VAN DeR Genugten, 1995, section 8.)

\section{Card counting systems for betting}

A card counting system is a vector $\varphi \in \mathbb{R}^{10}$ with the interpretation that card $j$ gets the score $\varphi_{j}, j=1, \ldots, 10$. During a rowgame a player cumulates the scores of all (C) VVS, 1997 


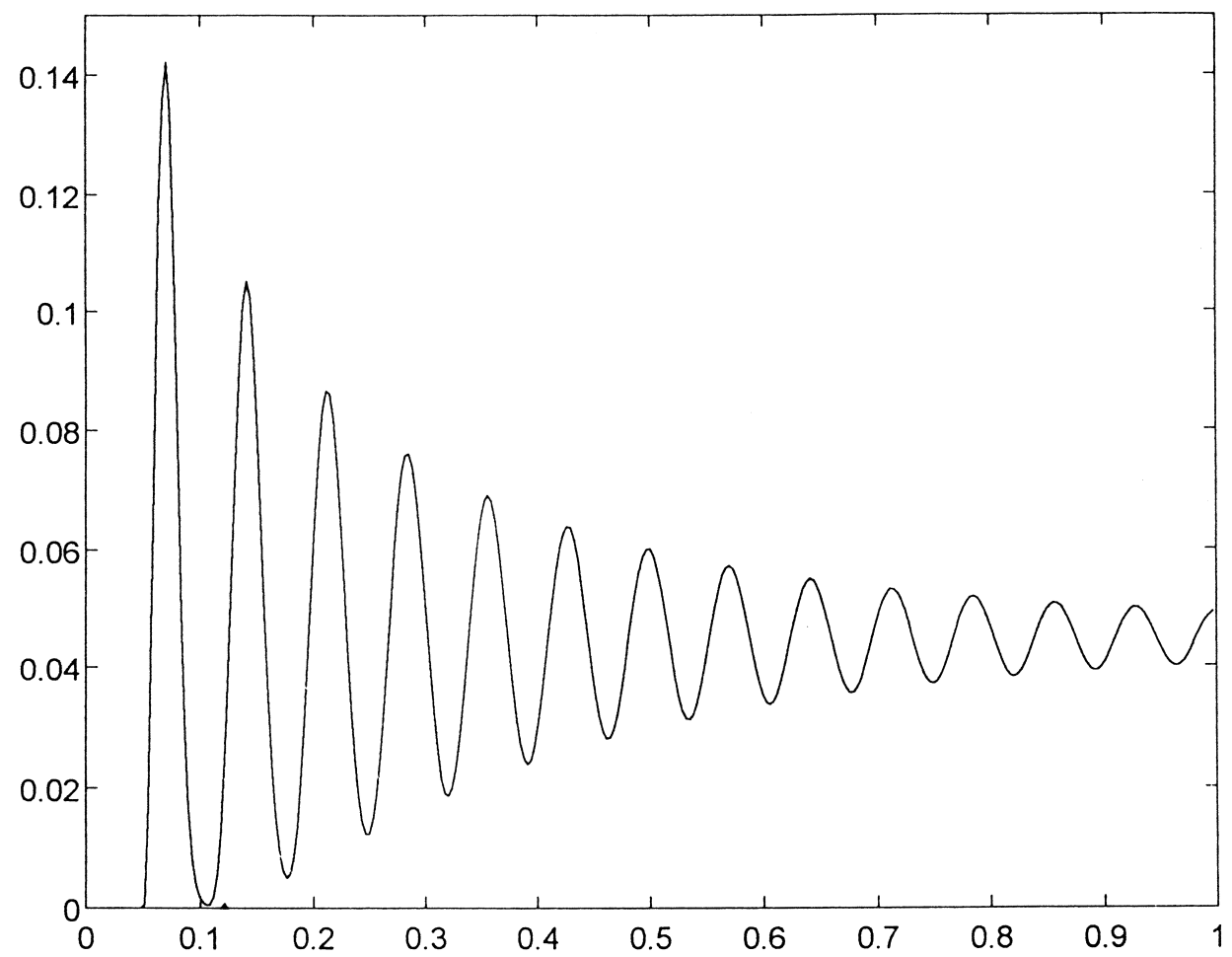

Fig. 9. Graphical representation of $p(t) / p(0)$ for $0<t<1\left(n=6, \lambda=1, a=7, S_{\text {bas }} ; p(0)=0.0690\right)$, ( $M=40,000,000$ rowgames).

successive dealt cards $X_{1}, X_{2}, \ldots$ using his counting system $\varphi$. This sum of scores is called the running count. So

$$
V(t)=\sum_{i=1}^{k t} \varphi_{x_{i}}
$$

is the running count up to a played card fraction $t$. The player makes his betting and playing decisions according to the true count $U(t)$, by definition the running count $V(t)$ divided by the number of remaining cards $k-k t$ in the shoe:

$$
U(t)=\frac{V(t)}{k(1-t)}=\sum_{j=1}^{10} \varphi_{j} F_{j}(t)
$$

where

$$
F_{j}(t)=\frac{c_{0}(j)-C(j)}{k-k t}
$$

denotes the played card fraction of card $j$ in a stock $C \in \mathcal{C}_{t}$. (c) VVS, 1997 
The basic idea behind the introduction of counting systems is that for large $n$ the conditional gain distribution $\mathcal{L}_{t}\left(G_{1}(C)\right)$ depends approximately only on the card fractions $F_{j}(t)$. In particular we can try to find a linear combination $U(t)$ such that

$$
\mathcal{L}_{t}\left(G_{1}(C)\right) \approx \mathcal{L}\left(g_{0}+U(t)\right)
$$

where $g_{0}$ is a norming constant, say

$$
g_{0}=E\left(G_{1}\left(c_{0}\right)\right)
$$

This is done by calculating $E\left(G_{1}(c)\right)$ for $c$ in a sufficiently large and widely spreadout set of stocks $\mathcal{C}_{\text {fit }}$. Then we take for $\varphi$ the LS-approximation in $\mathcal{C}_{\text {fit }}$. Often the restriction $\mu_{\varphi}=0$ for a centered system is added. Here $\mu_{\varphi}=\Sigma p_{j} \varphi_{j}$.

For the basic strategy $S_{\text {bas }}$ the LS-solution under the restriction $\mu_{\varphi}=0$ for a certain set $\mathcal{C}_{\text {fit }}$ is given by Table 10 .

Table 10. $\varphi$-values of $S_{\text {bas }}\left(g_{0}=-0.0061\right)$

\begin{tabular}{llcl}
\hline card $j$ & $\varphi_{j}$ & card $j$ & $\varphi_{j}$ \\
\hline 1 & -0.3411 & 6 & +0.2253 \\
2 & +0.1861 & 7 & +0.1154 \\
3 & +0.2153 & 8 & -0.0254 \\
4 & +0.2708 & 9 & -0.1006 \\
5 & +0.3451 & 10 & -0.2227 \\
\hline
\end{tabular}

The low cards $2-6$ exhibit an increasing pattern of $E\left(G_{1}(c)\right)$ with $F_{j}(t)$. So playing stocks containing few low cards are a disadvantage for players. For the high cards $7-10$ and $A=1$ we see that card 7 gives a slightly positive pattern, card 8 is neutral, card 9 is slightly negative and the cards $T=10$ and $A=1$ are strongly negative. So stocks rich with tens or aces are advantageous for players.

Now suppose that for a particular playing strategy a betfunction $H$ based on $\varphi$ is used, say

$$
H(c)=\bar{H}(U(t)), \quad c \in \mathcal{C}_{t}
$$

Then with (14):

$$
\mathcal{L}_{t}(G)=\mathcal{L}\left\{\bar{H}(U(t)) G_{1}(C)\right\} \approx \mathcal{L}\left\{\bar{H}(U(t))\left(g_{0}+U(t)\right)\right\}
$$

and, in particular,with (7)-(9):

$$
\mu_{G} \approx \sum_{t=0}^{\lambda-1 / k} p(t) E\left\{\bar{H}(U(t))\left(g_{0}+U(t)\right)\right\}
$$


It can be proved that $(U(t), 0<t<1)$ converges for $n \rightarrow \infty$ after standardization to the Brownian bridge. This leads to the following approximation of $U(t)$ for large $n$ :

$$
U(t) \stackrel{\mathcal{L}}{\approx} \frac{t}{1-t} \mu_{\varphi}+\sqrt{\frac{t}{1-t}} \frac{1}{\sqrt{k-1}} \sigma_{\varphi} Z
$$

with $\sigma_{\varphi}^{2}=\Sigma p_{j}\left(\varphi_{j}-\mu_{\varphi}\right)^{2}$ and $Z \sim N(0,1)$. The approximations (17)-(19) are very useful for analytic purposes. In particular, the corresponding optimal betfunction can be found approximately by maximizing the expectation on the right hand side of (18). This leads to $\bar{H}$ given by

$$
\bar{H}(U(t))= \begin{cases}1 & \text { if } U(t) \leq-g_{0} \\ B_{\max } & \text { if } U(t)>g_{0}\end{cases}
$$

For the corresponding maximal expected gain given $\left\{C \in \mathcal{C}_{\mathrm{t}}\right\}$ we get:

$$
E_{t}(G) \approx E\left\{\bar{H}(U(t))\left(g_{0}+U(t)\right)\right\}
$$

For a centered counting system $\left(\mu_{\varphi}=0\right)$ substitution of (19) into (21) leads after some calculations to

$$
\mu_{G} \approx g_{0}+\left(B_{\max }-1\right) \sum_{t=0}^{\lambda-1 / k} p(t)\left\{g_{0} Z_{0}\left(-g_{0} / g_{1}(t)\right)+g_{1}(t) Z_{1}\left(-g_{0} / g_{1}(t)\right)\right\}
$$

with

$$
\begin{aligned}
g_{1}(t) & =\sqrt{\frac{t}{1-t}} \frac{1}{\sqrt{k-1}} \sigma_{\varphi} \\
Z_{k}(t) & =\int_{t}^{\infty} \frac{1}{\sqrt{2 \pi}} u^{k} \mathrm{e}^{-1 / 2 u^{2}} \mathrm{~d} u, \quad k=0,1
\end{aligned}
$$

For the basic strategy $S_{\text {bas }}$ the corresponding approximating optimal strategy $\bar{H}_{\text {bas }}$ follows immediately from (20) with $\varphi$ in Table 22. The corresponding approximations for the expected gain follow from (22). Table 11 gives the results for the interesting values of $\lambda$ and $B_{\max }$. The table indicates clearly the influence of the cut card position $\lambda$ and the maximum bet $B_{\max }$.

Table 11. Expected gains $\mu_{\mathrm{G}}$ for $\left(\bar{H}_{\text {bas }}, S_{\text {bas }}\right)(n=6)$

\begin{tabular}{llllll}
\hline$B_{\max }$ & 1 & 2 & 25 & 37.5 & 50 \\
\hline$\lambda=\frac{1}{2}$ & -0.0061 & -0.0052 & 0.016 & 0.027 & 0.038 \\
$\lambda=\frac{2}{3}$ & -0.0061 & -0.0046 & 0.031 & 0.050 & 0.069 \\
\hline
\end{tabular}

Counting systems $\varphi$ based on LS are called theoretical because the scores $\varphi_{j}$ are not nice figures and therefore too complicated to use in practice. Therefore the next (C) VVS, 1997 
step is to replace $\varphi$ by a counting system $\psi$, obtained from $\varphi$ by rounding off. This leads to a practical running count

$$
S(t)=\sum_{i=1}^{k t} \psi_{X_{i}}
$$

and a practical true count

$$
T(t)=\frac{S(t)}{k-k t}=\sum_{j=1}^{10} \psi_{j} F_{j}(t)
$$

A betfunction based on the practical system $\psi$ is constructed from a betfunction based on the theoretical true count $\varphi$ through the LS-estimate $\hat{\varphi}$ of $\varphi$ based on $\psi$. We restrict ourselves to centered systems $\left(\mu_{\varphi}=\mu_{\psi}=0\right)$. Then

$$
\hat{\varphi}=\left(\rho_{\psi \varphi} \sigma_{\varphi} / \sigma_{\psi}\right) \psi
$$

where $\rho_{\psi \varphi}$ is the correlation coefficient between $\psi$ and $\varphi$ (i.e., $\rho_{\psi \varphi}=\sigma_{\psi \varphi} /\left(\sigma_{\varphi} \sigma_{\psi}\right)$ with $\left.\sigma_{\psi \varphi}=\Sigma p_{j}\left(\varphi_{j}-\mu_{\varphi}\right)\left(\psi_{j}-\mu_{\psi}\right)\right)$. With (12) and (16) this leads to the corresponding estimates

$$
\begin{aligned}
\hat{U}(t) & =\sum_{j=1}^{10} \hat{\varphi}_{j} F_{j}(t)=\rho_{\psi \varphi} \frac{\sigma_{\varphi}}{\sigma_{\psi}} T(t) \\
H(c) & =\bar{H}(\hat{U}(t))=\hat{H}(T(t)), \quad c \in \mathcal{C}_{t}
\end{aligned}
$$

The corresponding optimal betfunction $\hat{H}$ based on $T(t)$ follows from (20) and (28):

$$
\hat{H}(T(t))= \begin{cases}1 & \text { if } T(t) \leq t_{0} \\ B_{\max } & \text { if } T(t)>t_{0}\end{cases}
$$

where

$$
t_{0}=-g_{0} \frac{\sigma_{\psi}}{\rho_{\varphi \psi} \sigma_{\varphi}}
$$

The approximation of the corresponding expected gain is given by (22), where in the definition (23) of $g_{1}(t)$ we replace $\sigma_{\varphi}$ by $\rho_{\psi \varphi} \sigma_{\varphi}$ (in (22) the factor $\sigma_{\psi}$ cancels out). Therefore the rounding off procedure to get $\psi$ from $\varphi$ can be judged just on the base of the correlation coefficient! In literature $\rho_{\psi \varphi}$ is called the betting correlation.

Figure 12 gives the estimated $\mu_{\mathrm{G}}$ as a function of $\rho_{\phi \psi} \in(0.5,1)$ for $\lambda=2 / 3, n=6$ and interesting values of $B_{\max }$. The figure shows clearly the strong increase of the expected gain by using $\psi$-approximations of $\varphi$ with increasing correlation coefficient $\rho_{\varphi \psi}$.

The results of this section up till here can be generalized in several ways (higher dimensional count systems or side counting, non-linear approximations). We refer to VAN DeR Genugten (1995), sections 9 and 10 for a general setup. 


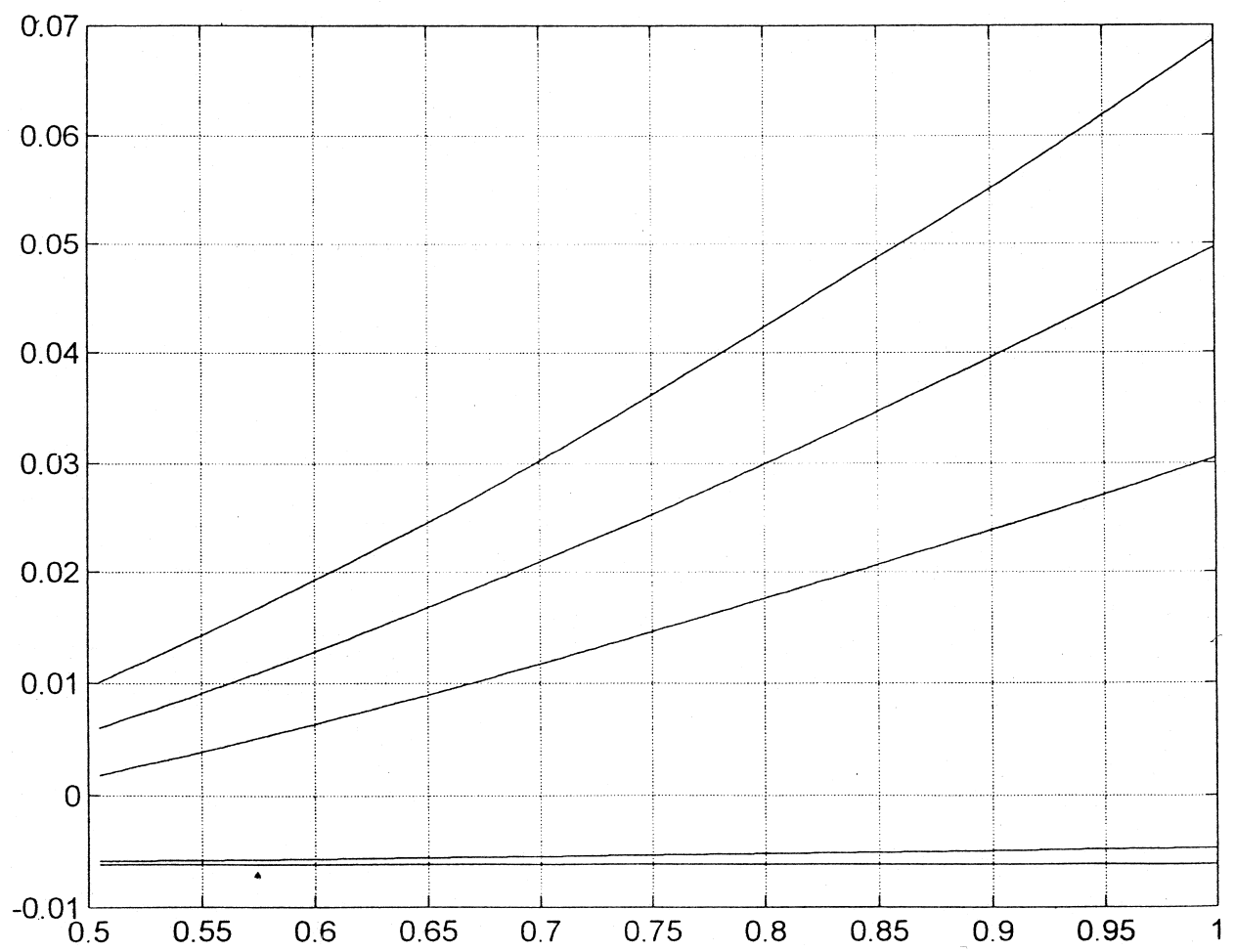

Fig. 12. Est. $\mu_{\mathrm{G}}$ for $S_{\text {bas }}$ as function of $\rho_{\varphi \psi}\left(B_{\max }=1,2,25,37.5\right.$ and $\left.50 ; \lambda=2 / 3, n=6\right)$.

We consider hereafter only two famous practical (centered) card counting systems: TTC (Thorp's Ten Count) and HiLo (High-Low). Table 13 gives the definition of both systems.

Table 13. $\psi$-values of TTC and HiLo

\begin{tabular}{lrlllllllll}
\hline card & 1 & 2 & 3 & 4 & 5 & 6 & 7 & 8 & 9 & 10 \\
\hline TTC & 4 & 4 & 4 & 4 & 4 & 4 & 4 & 4 & 4 & -9 \\
HiLo & -1 & 1 & 1 & 1 & 1 & 1 & 0 & 0 & 0 & -1 \\
\hline
\end{tabular}

The TTC-system is the most simple card counting system. Its true count $T=T(t)$ can be easily expressed in terms of the so-called $T$-ratio $T R=T R(t)$ of a stock: the number of non-tens divided by the number of tens:

$$
T=(9-4 T R) /(1+T R)
$$

For HiLo we use HL $=52 \times$ True Count.

In relation to the $\varphi$-values of the basic strategy, Table 14 follows from Tables 10 and 13 . 
Table 14. $(\varphi, \psi)$-values of counting systems with respect to $S_{\text {bas }}$

\begin{tabular}{llll}
\hline & $\rho_{\varphi \psi}$ & $t_{0}$ & \\
\hline TTC & 0.66 & +0.248 & $T R_{0}=2.06$ \\
HiLo & 0.96 & +0.0248 & $H L_{0}=1.3$ \\
\hline
\end{tabular}

This leads to the following expected gains in Table 15 for the betting strategies $\hat{H}_{\mathrm{TTC}}$ and $\hat{H}_{\text {HiLo }}$ based on (30) (the values of Table 11 are included for comparison).

Table 15. $\mu_{\mathrm{G}}$ of counting systems for $S_{\text {bas }}(n=6)$

\begin{tabular}{|c|c|c|c|c|c|c|}
\hline & $B_{\max }$ & 1 & 2 & 25 & 37.5 & 50 \\
\hline$\lambda=\frac{1}{2}$ & $\begin{array}{l}\hat{H}_{\mathrm{TTC}} \\
\hat{H}_{\text {HiLo }} \\
\bar{H}_{\text {bas }}\end{array}$ & $\begin{array}{l}-0.0061 \\
-0.0061 \\
-0.0061\end{array}$ & $\begin{array}{l}-0.0058 \\
-0.0053 \\
-0.0052\end{array}$ & $\begin{array}{l}0.0015 \\
0.014 \\
0.016\end{array}$ & $\begin{array}{l}0.0055 \\
0.024 \\
0.027\end{array}$ & $\begin{array}{l}0.010 \\
0.034 \\
0.038\end{array}$ \\
\hline$\lambda=\frac{2}{3}$ & $\begin{array}{l}\hat{H}_{\mathrm{TTC}} \\
\hat{H}_{\text {HiLo }} \\
\bar{H}_{\text {bas }}\end{array}$ & $\begin{array}{l}-0.0061 \\
-0.0061 \\
-0.0061\end{array}$ & $\begin{array}{l}-0.0055 \\
-0.0047 \\
-0.0046\end{array}$ & $\begin{array}{l}0.0092 \\
0.028 \\
0.031\end{array}$ & $\begin{array}{l}0.017 \\
0.046 \\
0.050\end{array}$ & $\begin{array}{l}0.025 \\
0.063 \\
0.069\end{array}$ \\
\hline
\end{tabular}

Clearly, the performance of the HiLo-system is much better than that of the TTC-system. However, TCC is much easier to use in practice.

As a final check on the estimations in Table 15 we performed a simulation for $\lambda=2 / 3, B_{\max }=50$ with the $\psi$-values of TTC and HiLo and the underlying $\varphi$ for $S_{\text {bas. }}$. Table 16 gives the results. Comparing Table 16 with $B_{\max }=50, \lambda=2 / 3$ in Table 15, we see a slight bias in the estimates. This bias follows a systematic pattern for varying $n$ (compare Section 7).

Table 16. Sim. gains of counting systems for $S_{\text {bas }}\left(n=6, \lambda=2 / 3, B_{\max }=50\right)(M=50,000,000$ rowgames $-\mu_{\mathrm{N}}=9.86$ )

\begin{tabular}{llll}
\hline betfunction & $\mu_{\mathrm{GR}}$ & $\pm 95 \%$ & $\mu_{\mathrm{G}}$ \\
\hline$\hat{H}_{\mathrm{TTC}}\left(T R_{0}=2.06\right)$ & 0.294 & \pm 0.02 & 0.0299 \\
$\hat{H}_{\mathrm{HiLo}}\left(H L_{0}=1.3\right)$ & 0.687 & \pm 0.02 & 0.0698 \\
$\bar{H}_{\text {bas }}\left(t_{0}=0.0061\right)$ & 0.733 & \pm 0.02 & 0.0744 \\
\hline
\end{tabular}

\section{Card counting systems for playing}

Card counting systems are also used for playing decisions. For BJHC the number of decks $n=6$ is large and therefore playing decisions different from the basic strategy can only increase the expected gain by a small amount. We only consider playing decisions for TTC and HiLo.

Table 17 gives the TTC-playing strategy $S_{\text {TTC }}$ and Table 18 the HiLo-playing strategy $S_{\text {HiLo }}$.

(For the construction we refer to VAN DER GenUGTEN, 1995, section 10.) We studied the effect of $S_{\text {bas }}, S_{\mathrm{TTC}}$ and $S_{\mathrm{HiLo}}$ in combination with various betfunctions. Based on (C) VVS, 1997 
Table 17. Playing strategy $S_{\text {TTC }}(T R=$ Ten Ratio $)$

INSURANCE: insure if $T R \leq 2$

SPLITTING (Split if $T R \leq$, if underlined then split it $T R \geq$ )

\begin{tabular}{lllllllllll}
\hline Dealercard Pair & A & 2 & 3 & 4 & 5 & 6 & 7 & 8 & 9 & T \\
\hline AA & 1.4 & 4.2 & 4.5 & 4.7 & 5.0 & 5.3 & 4.3 & 3.9 & 3.7 & 3.2 \\
22 & 0 & 3.0 & 3.7 & $\infty$ & $\infty$ & $\infty$ & $\infty$ & $\underline{2.6}$ & $\underline{8.0}$ & 0 \\
33 & 0 & 2.3 & $\infty$ & $\infty$ & $\infty$ & $\infty$ & $\infty$ & $\underline{2.4}$ & $\underline{5.5}$ & 0 \\
44 & 0 & 1.4 & 1.6 & 1.9 & 2.3 & 2.4 & $\underline{6.2}$ & $\underline{7.0}$ & 0 & 0 \\
55 & 0 & 0 & 0 & 0 & 0 & 0 & 0 & 0 & 0 & 0 \\
66 & 0 & 2.4 & 2.7 & 3.0 & 3.3 & 3.7 & 0 & 0 & 0 & 0 \\
77 & 0 & 3.6 & 4.1 & 4.8 & 5.8 & $\infty$ & $\infty$ & 2.1 & 0 & 0 \\
777 & 0 & 3.0 & 3.3 & 3.7 & 4.2 & 5.3 & 4.9 & 0 & 0 & 0 \\
88 & 0 & $\infty$ & $\infty$ & $\infty$ & $\infty$ & $\infty$ & $\infty$ & $\infty$ & $\infty$ & $\underline{2.8}$ \\
99 & 0 & 2.6 & 2.9 & 3.1 & 3.5 & 3.4 & 1.8 & 4.1 & 4.1 & 0 \\
TT & 0 & 1.4 & 1.5 & 1.6 & 1.7 & 1.8 & 0 & 0 & 0 & 0 \\
\hline
\end{tabular}

DOUBLE DOWN (DDown if $T R \leq$ )

\begin{tabular}{lllllllllll}
\hline Dealercard Sum & A & 2 & 3 & 4 & 5 & 6 & 7 & 8 & 9 & T \\
\hline H9 & 0 & 2.2 & 2.4 & 2.6 & 2.9 & 3.2 & 1.8 & 1.5 & 0 & 0 \\
H10 & 0 & 3.6 & 3.9 & 4.2 & 4.8 & 5.1 & 3.5 & 3.0 & 2.5 & 0 \\
H11 & 0 & 3.9 & 4.1 & 4.5 & 5.0 & 5.4 & 3.9 & 3.3 & 2.8 & 2.1 \\
S19 & 0 & 1.2 & 1.3 & 1.4 & 1.5 & 1.6 & 0 & 0 & 0 & 0 \\
S20 & 0 & 1.4 & 1.5 & 1.6 & 1.7 & 1.8 & 0 & 0 & 0 & 0 \\
S21 & 0 & 0 & 0 & 0 & 1.0 & 1.0 & 0 & 0 & 0 & 0 \\
\hline
\end{tabular}

DRAW/STAND (Draw if $T R \geq$ )

\begin{tabular}{|c|c|c|c|c|c|c|c|c|c|c|}
\hline Dealercard Sum & A & 2 & 3 & 4 & 5 & 6 & 7 & 8 & 9 & $\mathrm{~T}$ \\
\hline H12 & 0 & 2.0 & 2.2 & 2.3 & 2.4 & 2.3 & 0 & 0 & 0 & 1.1 \\
\hline H13 & 1.2 & 2.3 & 2.5 & 2.7 & 2.9 & 2.8 & 0 & 0 & 0 & 1.3 \\
\hline H14 & 1.3 & 2.7 & 3.0 & 3.2 & 3.5 & 3.3 & 0 & 0 & 1.2 & 1.6 \\
\hline 777 & 0 & 2.2 & 2.4 & 2.5 & 2.7 & 2.7 & 0 & 0 & 0 & 0 \\
\hline H15 & 1.4 & 3.2 & 3.5 & 3.9 & 4.3 & 4.1 & 0 & 0 & 1.4 & 1.9 \\
\hline H16 & 1.5 & 3.8 & 4.2 & 4.7 & 5.4 & 5.0 & 0 & 1.4 & 1.8 & 2.2 \\
\hline H17 & 3.0 & $\infty$ & $\infty$ & $\infty$ & $\infty$ & $\infty$ & $\infty$ & $\infty$ & $\infty$ & $\infty$ \\
\hline H18 & $\infty$ & $\infty$ & $\infty$ & $\infty$ & $\infty$ & $\infty$ & $\infty$ & $\infty$ & $\infty$ & $\infty$ \\
\hline S17 & 0 & 0 & 0 & 0 & 0 & 0 & 0 & 0 & 0 & 0 \\
\hline $\mathrm{S} 18$ & 2.1 & 5.1 & 5.5 & 6.2 & 6.6 & $\infty$ & $\infty$ & 5.1 & 0 & 0 \\
\hline S19 & $\infty$ & $\infty$ & $\infty$ & $\infty$ & $\infty$ & $\infty$ & $\infty$ & $\infty$ & $\infty$ & $\infty$ \\
\hline
\end{tabular}

Table 14 and some further simulations with $S_{\text {TTC }}$ and $S_{\text {HiLo }}$ we took the (modified) simple bounds of Table 19.

The betfunctions always take the maximum bet $B_{\max }$ for appropriate values of $T R$ and $H L$ respectively. This leads to a large variance in the gain. So these strategies can only be played in practice by high budget players with a large starting capital. For low budget players with a low (or moderate) capital other betfunctions come into view. Therefore we consider also two low budget betfunctions $\tilde{H}_{\mathrm{TTC}}$ and $\tilde{H}_{\text {HiLo }}$ specified in (C) VVS, 1997 
Table 18. Playing strategy $S_{\mathrm{HiLo}}(H L=52 \times$ true count $)$

INSURANCE: insure if $H L \geq 3$

SPLITTING (Split if $H L>$ )

\begin{tabular}{|c|c|c|c|c|c|c|c|c|c|c|}
\hline Dealercard Pair & A & 2 & 3 & 4 & 5 & 6 & 7 & 8 & 9 & $\mathrm{~T}$ \\
\hline AA & $+\infty$ & $-\infty$ & $-\infty$ & $-\infty$ & $-\infty$ & $-\infty$ & $-\infty$ & $-\infty$ & $-\infty$ & -9 \\
\hline 22 & $+\infty$ & $-3 \frac{1}{2}$ & -6 & -8 & $-9 \frac{1}{2}$ & $-\infty$ & $-\infty$ & $+4 \frac{1}{2}$ & $+\infty$ & $+\infty$ \\
\hline 33 & $+\infty$ & 0 & -5 & -8 & $-9 \frac{1}{2}$ & $-\infty$ & $-\infty$ & $+5 \frac{1}{2}$ & $+\infty$ & $+\infty$ \\
\hline 44 & $+\infty$ & $+\infty$ & +8 & +3 & $-\frac{1}{2}$ & $-1 \frac{1}{2}$ & $+\infty$ & $+\infty$ & $+\infty$ & $+\infty$ \\
\hline 55 & $+\infty$ & $+\infty$ & $+\infty$ & $+\infty$ & $+\infty$ & $+\infty$ & $+\infty$ & $+\infty$ & $+\infty$ & $+\infty$ \\
\hline 66 & $+\infty$ & -2 & $-4 \frac{1}{2}$ & $-6 \frac{1}{2}$ & $-8 \frac{1}{2}$ & $-\infty$ & $+\infty$ & $+\infty$ & $+\infty$ & $+\infty$ \\
\hline $7(7) 7$ & $+\infty$ & -7 & $-8 \frac{1}{2}$ & $-\infty$ & $-\infty$ & $-\infty$ & $-\infty$ & $+\infty$ & $+\infty$ & $+\infty$ \\
\hline 88 & $+\infty$ & $-\infty$ & $-\infty$ & $-\infty$ & $-\infty$ & $-\infty$ & $-\infty$ & $-\infty$ & $-\infty$ & $+\infty$ \\
\hline 99 & $+\infty$ & -3 & $-4 \frac{1}{2}$ & $-5 \frac{1}{2}$ & -7 & $-7 \frac{1}{2}$ & +3 & -9 & $-\infty$ & $+\infty$ \\
\hline TT & $+\infty$ & $+\infty$ & $+8 \frac{1}{2}$ & $+6 \frac{1}{2}$ & +5 & $+4 \frac{1}{2}$ & $+\infty$ & $+\infty$ & $+\infty$ & $+\infty$ \\
\hline
\end{tabular}

DOUBLE DOWN (DDown if $H L>$ )

\begin{tabular}{|c|c|c|c|c|c|c|c|c|c|c|}
\hline Dealercard Sum & A & 2 & 3 & 4 & 5 & 6 & 7 & 8 & 9 & $\mathrm{~T}$ \\
\hline H9 & $+\infty$ & +1 & -1 & -3 & $-4 \frac{1}{2}$ & $-\frac{1}{2}$ & $+3 \frac{1}{2}$ & $+7 \frac{1}{2}$ & $+\infty$ & $+\infty$ \\
\hline $\mathrm{H} 10$ & $+\infty$ & -9 & $-\infty$ & $-\infty$ & $-\infty$ & $-\infty$ & $-6 \frac{1}{2}$ & $-4 \frac{1}{2}$ & $-1 \frac{1}{2}$ & $+\infty$ \\
\hline H11 & $+\infty$ & $-\infty$ & $-\infty$ & $-\infty$ & $-\infty$ & $-\infty$ & $-\infty$ & $-7 \frac{1}{2}$ & -5 & $+3 \frac{1}{2}$ \\
\hline S19 & $+\infty$ & $+\infty$ & $+\infty$ & +9 & $+6 \frac{1}{2}$ & +7 & $+\infty$ & $+\infty$ & $+\infty$ & $+\infty$ \\
\hline S20 & $+\infty$ & $+\infty$ & $+8 \frac{1}{2}$ & $+6 \frac{1}{2}$ & +5 & $+4 \frac{1}{2}$ & $+\infty$ & $+\infty$ & $+\infty$ & $+\infty$ \\
\hline S21 & $+\infty$ & $+\infty$ & $+\infty$ & $+\infty$ & +8 & +8 & $+\infty$ & $+\infty$ & $+\infty$ & $+\infty$ \\
\hline
\end{tabular}

DRAW/STAND (Draw if $H L \leq)$

\begin{tabular}{|c|c|c|c|c|c|c|c|c|c|c|}
\hline Dealercard Sum & A & 2 & 3 & 4 & 5 & 6 & 7 & 8 & 9 & $\mathrm{~T}$ \\
\hline $\mathrm{H} 12$ & $+\infty$ & +3 & $+1 \frac{1}{2}$ & 0 & $-1 \frac{1}{2}$ & -1 & $+\infty$ & $+\infty$ & $+\infty$ & $+\infty$ \\
\hline H13 & $+\infty$ & -1 & $-2 \frac{1}{2}$ & -4 & $-5 \frac{1}{2}$ & $-5 \frac{1}{2}$ & $+\infty$ & $+\infty$ & $+\infty$ & $+\infty$ \\
\hline H14(777) & $+\infty$ & -4 & -5 & $-6 \frac{1}{2}$ & -8 & $-8 \frac{1}{2}$ & $+\infty$ & $+\infty$ & $+\infty$ & $+\infty$ \\
\hline H15 & $+9 \frac{1}{2}$ & -6 & -7 & $-8 \frac{1}{2}$ & $-9 \frac{1}{2}$ & $-\infty$ & $+\infty$ & $+\infty$ & +8 & $+4 \frac{1}{2}$ \\
\hline H16 & $+8 \frac{1}{2}$ & $-9 \frac{1}{2}$ & $-\infty$ & $-\infty$ & $-\infty$ & $-\infty$ & +8 & +7 & $+4 \frac{1}{2}$ & 0 \\
\hline H17 & $-6 \frac{1}{2}$ & $-\infty$ & $-\infty$ & $-\infty$ & $-\infty$ & $-\infty$ & $-\infty$ & $-\infty$ & $-\infty$ & $-\infty$ \\
\hline H18 & $-\infty$ & $-\infty$ & $-\infty$ & $-\infty$ & $-\infty$ & $-\infty$ & $-\infty$ & $-\infty$ & $-\infty$ & $-\infty$ \\
\hline S17 & $+\infty$ & $+\infty$ & $+\infty$ & $+\infty$ & $+\infty$ & $+\infty$ & $+\infty$ & $+\infty$ & $+\infty$ & $+\infty$ \\
\hline S18 & +1 & $-\infty$ & $-\infty$ & $-\infty$ & $-\infty$ & $-\infty$ & $-\infty$ & $-\infty$ & $+\infty$ & $+\infty$ \\
\hline S19 & $-\infty$ & $-\infty$ & $-\infty$ & $-\infty$ & $-\infty$ & $-\infty$ & $-\infty$ & $-\infty$ & $-\infty$ & $-\infty$ \\
\hline
\end{tabular}

Table 20 . The betting strategy $\tilde{H}_{\mathrm{TTC}}$ aims to minimize the probability of ruin starting with a moderate capital.

According to WIND and WIND (1994) the betting strategy $\tilde{H}_{\text {HiLo }}$ follows from a conservative interpretation of the Kelly-principle to choose the bet in such a way that

Table 19. Modified $(\psi, \varphi)$-values w.r.t. $S_{\text {bas }}$

\begin{tabular}{lll}
\hline & $t_{0}$ & \\
\hline TTC & +0.194 & $T R_{0}=2.1$ \\
HiLo & +0.0288 & $H L_{0}=1.3$ \\
\hline
\end{tabular}

(C) VVS, 1997 
Table 20. Betting of low and high budget players $\left(B_{\max }=50\right)$

\begin{tabular}{lcclcc}
\hline & \multicolumn{2}{c}{ TTC } & & \multicolumn{2}{c}{ HiLo } \\
\cline { 2 - 5 } Class & $\begin{array}{l}\hat{H}_{\text {TTC }} \\
\text { (High) }\end{array}$ & $\begin{array}{l}\tilde{H}_{\text {TTC }} \\
(\text { Low })\end{array}$ & Class & $\begin{array}{c}\hat{H}_{\text {HiLo }} \\
(\text { High })\end{array}$ & $\begin{array}{c}\tilde{H}_{\text {HiLo }} \\
\text { (Low) }\end{array}$ \\
\hline$T R>2.3$ & 1 & 1 & $H L<1 / 2$ & 1 & 1 \\
$2.1<T R \leq 2.3$ & 1 & 1 & $1 / 2 \leq H L<1$ & 1 & 1 \\
$2.0<T R \leq 2.1$ & 50 & 2 & $1 \leq H L<2$ & 50 & 5 \\
$1.9<T R \leq 2.0$ & 50 & 6 & $2 \leq H L<3$ & 50 & 10 \\
$1.8<T R \leq 1.9$ & 50 & 9 & $3 \leq H L<4$ & 50 & 15 \\
$1.7<T R \leq 1.8$ & 50 & 13 & $4 \leq H L<5$ & 50 & 20 \\
$T R \leq 1.7$ & 50 & 21 & $H L \geq 5$ & 50 & 25 \\
\hline
\end{tabular}

Table 21. Simulated gains $\left(n=6, a=7, \lambda=2 / 3, B_{\max }=50\right)$

$\left(M=50,000,000\right.$ rowgames $-\mu_{\mathrm{N}}=9.98$ games $)$

\begin{tabular}{llllll}
\hline Strategy & $\mu_{\mathrm{GR}}$ & $\sigma_{\mathrm{GR}}$ & $\pm 95 \% C I$ & $\mu_{\mathrm{G}}$ & $\mu_{\mathrm{B}}$ \\
\hline$\left(\hat{H}_{\mathrm{HiLo}}, S_{\mathrm{HiLo}}\right)$ & +0.918 & 87.7 & 0.027 & +0.0931 & 14.7 \\
$\left(\hat{H}_{\mathrm{HiLo}}, S_{\text {bas }}\right)$ & +0.702 & 86.5 & 0.027 & +0.0711 & 14.4 \\
$\left(\hat{H}_{\mathrm{TTC}}, S_{\mathrm{TTC}}\right)$ & +0.569 & 75.5 & 0.023 & +0.0576 & 11.2 \\
$\left(\hat{H}_{\mathrm{TTC}}, S_{\text {bas }}\right)$ & +0.321 & 73.8 & 0.023 & +0.0326 & 10.8 \\
$\left(\tilde{H}_{\mathrm{HiLo}}, S_{\mathrm{HiLo}}\right)$ & +0.240 & 20.9 & 0.006 & +0.0243 & 3.60 \\
$\left(\tilde{H}_{\mathrm{HiLo}}, S_{\text {bas }}\right)$ & +0.171 & 20.1 & 0.006 & +0.0174 & 3.51 \\
$\left(\tilde{H}_{\mathrm{TTC}}, S_{\mathrm{TTC}}\right)$ & +0.062 & 11.2 & 0.003 & +0.0063 & 2.02 \\
$\left(\tilde{H}_{\mathrm{TTC}}, S_{\text {bas }}\right)$ & +0.009 & 10.7 & 0.003 & +0.0009 & 1.97 \\
$\left(H \equiv 1, S_{\mathrm{TTC}}\right)$ & -0.0425 & 3.51 & 0.0011 & -0.0043 & 1.11 \\
$\left(H \equiv 1, S_{\mathrm{HiLo}}\right)$ & -0.0456 & 3.50 & 0.0011 & -0.0046 & 1.10 \\
$\left(H \equiv 1, S_{\text {bas }}\right)$ & -0.0524 & 3.50 & 0.0011 & -0.0053 & 1.10 \\
\hline
\end{tabular}

the expected growth of one's capital is maximized. For details we refer to VAN DER GenUGTen (1995), section 10.

We made several simulation runs to obtain the performance of these strategies. Table 21 gives an overview. This table contains also the standard deviation $\sigma_{\mathrm{GR}}$ of one rowgame and the mean bet $\mu_{\mathrm{B}}$ of one game. We see that the HiLo-system is better (but also more complicated) than the TTC-system. There is a large difference between the high-budget systems $\hat{H}$ and the low-budget systems $\tilde{H}$. However, even low budget players can beat the dealer in the long run!

\section{References}

Baldwin, R., W. Cantey, H. Maisel and J. MacDermott (1956), The optimal strategy in Blackjack, Journal of the American Statistical Association 51, 419-439.

BillingsLy, P. (1968), Convergence of probability measures, Wiley, New York.

BLACK, J. (1993), The money-spinners: how professional gamblers beat the casinos at their own game, Faber and Faber Ltd., Leicester.

Bond, N. A. (1974), Basic strategy and expectation in Casino Blackjack, Organizational behavior and human performance 12, 413-428.

Chambliss, C. R. and T. C. Roginski (1990), Fundamentals of Blackjack, Gambler's Book Club Press, Las Vegas. 
Chau, A. W. and J. G. Phillips (1995), Effects of perceived control upon wagering and attributions in computer blackjack, Journal of General Psychology 122, 253-269.

Dalton, M. (1993), Blackjack: a professional reference, 3rd edit., Spur of the Moment Publishing, Merritt Island.

Epstein, R. A. (1977), The theory of gambling and statistical logic, Academic Press, New York. GotTlieb, G. (1985), An analytic derivation of Blackjack win rates, Operations Research 33, 971-981.

Griffin, P. A. (1988), The theory of Blackjack, Huntington Press, Las Vegas.

KerEn, G. and W. A. WAgenaAR (1985), On the psychology of playing blackjack: Normative and descriptive considerations with implications for decision theory, Journal of Experimental Psychology, General 114, 133-158.

THorp, E. O. (1960), Fortune's formula: the game of Blackjack, Notices American Mathematical Society, 935-936.

Thorp, E. O. (1966), Beat the dealer, 2nd edit, Vintage books, New York.

VAn Der Genugten, B. B. (1993), Blackjack in Holland Casino's: hoe de dealer te verslaan, Tilburg University Press, Tilburg (in Dutch).

VAN DER Genugten, B. B. (1995), Blackjack in Holland Casino's: basic, optimal and winning strategies, Research Memorandum FEW 711, Tilburg University, Tilburg.

WAgEnAaR, W. A. (1988), Paradoxes of gambling behavior, East Essex, Erlbaum.

WIND, M. A. and M. M. WIND (1994), Winnen met Black Jack, Gottmer/Becht, Bloemendaal (in Dutch).

YAKowitz, S. and M. Kollier (1992), Machine learning for optimal blackjack counting strategies, Journal of Statistical Planning and Inference 33, 295-309.

Received: April 1993. Revised October 1996. 